\title{
Geometric representation of interval exchange maps over algebraic number fields
}

\author{
G. Poggiaspalla, J. H. Lowenstein†, and F. Vivaldi \\ School of Mathematical Sciences, Queen Mary, University of London, London E1 4NS, UK \\ $\dagger$ †ept. of Physics, New York University, 2 Washington Place, New York, NY 10003, USA
}

\begin{abstract}
This paper is concerned with the restriction of interval exchange transformations to algebraic number fields, which leads to maps on lattices. We characterize renormalizability arithmetically, and study its relationships with a geometrical quantity that we call the drift vector. We exhibit some examples of renormalizable IET with zero and non-zero drift vector and carry out some investigations of their properties. In particular we look for evidence of the finite decomposition property on a family of IETs extending the example studied in [18].
\end{abstract}

November 11, 2018

\section{Introduction}

An interval exchange transformations (IET) is a one-dimensional map $E$ of an interval, consisting of a rearrangement of a partition of that interval. Let $\left\{\Omega_{i}\right\}$ be an $N$-elements partition of $\Omega=[0,1)$, and let $\pi$ be a permutation of the integers $1, \ldots, N$. We define $E$ as the map that rearranges the intervals according to the permutation $\pi$, preserving orientation. This procedure defines $N$ translation vectors $\tau_{i}$

$$
E(x)=x+\tau_{i} \quad x \in \Omega_{i} .
$$

We assume that this permutation is irreducible, that is, $\pi\{1, \ldots, k\}=\{1, \ldots, k\}$ if and only if $k=N$.

There is substantial literature on the subject. An early theorem of Keane [13] states that if the permutation is irreducible and the set of lengths rationally independent, then 
two discontinuity points never lie on the same orbit and $E$ is minimal. A stronger results was later obtained by Masur and by Veech [20,25]: given an irreducible permutation, for almost all set of lengths, the associated interval exchange map is minimal and uniquely ergodic.

Boshernitzan [8] gave the following ergodicity criterion: if we let $\epsilon_{k}$ be the shortest length of an interval of the partition of $E^{k}$, then if

$$
\lim _{k \rightarrow \infty} k \epsilon_{k}>0
$$

$E$ is uniquely ergodic. A result of Katok [12] ensures that interval exchanges cannot be mixing. However, a recent result by Avila and Forni [3] shows that, for any permutation which is not a rotation (meaning that $\pi(i) \not \equiv i+1(\bmod N))$, the interval exchange map associated to Lebesgue-almost all set of lengths is weakly-mixing.

Here we consider a uniquely ergodic interval exchange map $E$, and we assume that the lengths of the sub-intervals $\Omega_{i}$ generate an algebraic extension $K$ of degree $n$ of the rationals. The module of $E$ is the set of all linear integral combinations of the sub-interval lengths, namely

$$
\mathcal{M}=\left\{\sum_{i=1}^{N} m_{i}\left|\Omega_{i}\right|: m_{i} \in \mathbb{Z}\right\}
$$

and the rank of $E$ is the rank of its module. Algebraic IETs constitute a countable, hence exceptional, family. Little is known about these maps, the only substantial result being the Boshernitzan and Carroll theorem [9]: if an IET is defined over a quadratic field, then, up to rescaling, the number of induced maps on sub-intervals is finite. In short, quadratic IETs can be renormalized. In general, interval exchanges over algebraic number fields of higher degree do not have this nice property. If they do, then their rank must be greater than two.

The code $\omega=\omega(x)$ of a point $x \in \Omega$ is constructed with respect to the natural partition

$$
\omega_{j}=i \quad \Leftrightarrow \quad E^{j-1} x \in \Omega_{i} .
$$

Let $e_{1}, \ldots, e_{N}$ be the canonical basis of $\mathbb{Z}^{N}$. We associate to each word $\omega$ its sub-word $\omega^{(k)}$ of length $k$, and the sequence $s\left(\omega^{(k)}\right)$ in $\mathbb{Z}^{N}$ defined as follows

$$
s\left(\omega^{(k)}\right)=\sum_{j=1}^{k} e_{\omega_{j}} \quad k=1,2, \ldots
$$

We call it the staircase function. If we denote by $\left|\omega^{(k)}\right|_{i}$ the number of occurrences of $i$ in $\omega^{(k)}$, then we have

$$
s\left(\omega^{(k)}\right)=\sum_{i=1}^{N}\left|\omega^{(k)}\right|_{i} e_{i} .
$$

This expression may be rewritten as follows

$$
s\left(\omega^{(k)}\right)=k \Lambda+D_{k} .
$$


Here $\Lambda$ is the vector of the lengths of the intervals

$$
\Lambda=\left(\left|\Omega_{1}\right|, \ldots,\left|\Omega_{N}\right|\right)
$$

and $D_{k}$ is the vector of the local discrepancies of the sequence $\omega$ in each cylinder $(i)$ or, equivalently, of the sequence $\left(E^{k} x\right)$ in each intervals $\Omega_{i}$

$$
D_{k}=\left(D_{k}\left(x, \Omega_{1}\right), \ldots, D_{k}\left(x, \Omega_{N}\right)\right)
$$

and

$$
D_{k}\left(x, \Omega_{i}\right)=\sum_{j=1}^{k} \mathbf{1}_{\Omega_{i}}\left(E^{j=1} x\right)-k\left|\Omega_{i}\right|=\left|\omega^{(k)}\right|_{i}-k\left|\Omega_{i}\right|,
$$

where $\mathbf{1}_{\Omega_{i}}$ is the characteristic function of the interval $\Omega_{i}$. By Birkhoff's ergodic theorem and using minimality we have $\left\|D_{k}\right\|=o(k)$; the staircase of each point thus has the principal direction $\Lambda$ with sub-linear perturbations.

The theory of the deviation from the ergodic averages in IET is closely related to the theory of geodesic flows on flat-surfaces, for which strong results have been given by Zorich [27,28]. Specifically, we suppose that the IET can be described as a section of a geodesic flow on a flat surface of genus $g$. Then, for almost all such IETs, there is a nested sequence of subspaces

$$
\mathcal{H}_{1} \subset \mathcal{H}_{2} \subset \cdots \subset \mathcal{H}_{g} \subset \mathbb{R}^{N}
$$

with $\mathcal{H}_{1}$ being the one-dimensional subspace spanned by $\Lambda$, and each $\mathcal{H}_{i}$ being $i$-dimensional, such that, for any $f \in \mathcal{H}_{i}^{\perp}$ with $\|f\|=1$

$$
\limsup _{k \rightarrow \infty} \frac{\log \left\langle s\left(\omega^{(k)}\right), f\right\rangle}{\log k}=\nu_{i}
$$

(The symbol $\langle$,$\rangle denotes the standard inner product.) This result says that the deviations$ of the staircase function from the ergodic means follow some power laws, the exponents of which are given by the Lyapunov exponents $\nu_{i}$, depending only on the so-called Rauzy class the IET belongs to.

The aim of this paper to introduce a lattice representation of algebraic IETs, following a method developed for higher dimensional piecewise isometries [14,16], and analogous to the representation used in [18]. Since the translations $\tau_{i}$ are expressed as integral linear combinations of the lengths, for any point $\xi$ in $K \cap[0,1)$ the set $\xi+\mathcal{M}$ is invariant under $E$ and contains the orbit of $\xi$. We then identify $\xi+\mathcal{M}$ with the lattice $\mathbf{L}_{\xi} \cong \mathbb{Z}^{m}$, where $m \leq n$ is the rank of $\mathcal{M}$. This construction may be seen as a projection of the lattice $\mathbb{Z}^{N}$ in which the staircase is built onto the lattice $\mathbf{L}$ where the dynamics takes place. In this way we obtain a discrete geometric representation of the IET, restricted to a dense set of algebraic points.

The content of this paper is the following:

In section 2 we introduce a module-theoretic description of an algebraic IET and define a fundamental dynamical invariant, the drift vector $\mathcal{S}$. We then show that the drift vector is a representation of the classical Sah-Arnoux-Fathi invariant (proposition 5). Most of 
the constructs developed in this section refer to the case of maximal rank; this restriction is justified by the fact that renormalizable IETs have this property (see theorem 1 below).

In section 3 we deal with renormalizability of algebraic IETs and we describe the role of the drift vector in the renormalization process. We then prove the main result of this section, which appears as theorem [6 and proposition 8. (For background on substitution dynamical systems see [22].)

Theorem 1 Let $E$ be a uniquely ergodic interval exchange transformation on $N$ intervals, which is renormalizable with scaling ratio $\rho$. Then $\rho$ is a unit in the ring $\mathbb{Z}[\beta]$, where $\beta=\rho^{-1}$ is the largest eigenvalue of the incidence matrix of the primitive substitution associated with $E$. The map $E$ is defined over $\mathbb{Q}(\rho)$, its rank $n$ is maximal and equal to the degree of $\rho$, and

(i) if the drift vector is non-zero, then $n$ is even and $n \leq N$;

(ii) if the drift vector is zero, then $2 n \leq N$.

Subsequently, we introduce the recursive tiling property and formalism (Vershik coding), and give estimates for the asymptotic behaviour of points with eventually periodic code in the scaling dynamics (propositions 9 and 11). We also give a condition for these points to be of positive density on the lattice (proposition 10).

Section 4 is devoted to the analysis of specific examples. Using Rauzy induction, we construct a renormalizable IET of degree four over four intervals, with non-zero drift vector. We then construct an infinite family of cubic renormalizable IET with zero driftvector, which belong to the so-called Arnoux-Rauzy family [6]. We also perform numerical investigations which give strong evidences that some of these examples share the finite decomposition property encountered in [18]. This property, which we establish in one case (proposition 13) is described as follows. The field $K$ may be represented as the disjoint union of invariant layers $\xi+\mathcal{M}$, for a suitable set of representative points $\xi \in K$. An algebraic IET has the finite decomposition property if the restriction of each layer to the unit interval is the union of finitely many orbits.

These results show connections between the finite decomposition property of a renormalizable IET and the Pisot property of the (reciprocal of the) scaling constant. This problem deserves further investigation.

Acknowledgements. This research was supported by EPSRC grant No GR/S62802/01. One of the authors (GP) wants to thank S. Ferenczi, X. Bressaud and P. Arnoux for some enlightening discussions. 


\section{Lattice dynamics and drift vector}

In this section we consider algebraic IETs with maximal rank, for which an interesting arithmetical theory arises. In the next section we shall see that renormalizable IETs have this property.

\section{$2.1 \quad$ Lattices}

We choose a basis for $\mathcal{M}$ as a $\mathbb{Z}$-module, and consider the translation vectors $\tau_{i} \in \mathcal{M}$ defined by $E$-cf. equation (1). Let $\phi$ be the map from $\mathcal{M}$ in $\mathbf{L}$ sending the basis elements of $\mathcal{M}$ onto the canonical basis of $\mathbf{L}$. We have two lattices, one is the symbolic lattice $\mathbb{Z}^{N}$ in which the staircase was defined; the other is $\mathbf{L}$, it has dimension $n$ and represents the module in which the dynamics takes place. We call $\pi$ the projection from the former in the latter; this is a linear map such that

$$
\pi\left(e_{i}\right)=v_{i} \quad v_{i}:=\phi\left(\tau_{i}\right)
$$

which is represented by an $n \times N$ matrix. We denote by $\psi=\phi \circ E \circ \phi^{-1}$ the map conjugate to $E$ on the lattice $\mathbf{L}$.

As we have a map of the unit interval, the dynamics of $\psi$ does not take place on all of $\mathbf{L}$, but only on the invariant slab of points $z \in \mathbf{L}$ such that $\phi^{-1}(z) \in[0,1)$. We are then led to consider the set $\mathcal{M} \cap[0,1)$ for which we require a suitable representation. Let

$$
\mathcal{O}=\{\zeta \in K: \zeta \mathcal{M} \subset \mathcal{M}\}
$$

where $K$ is the smallest field containing $\mathcal{M}$. Then $\mathcal{O}$ is an order 11 in $K$. Let $\nu_{1}, \ldots, \nu_{n}$ be a $\mathbb{Z}$-basis for $\mathcal{M}$, and let $d$ be the smallest positive integer such that $d \nu_{k} \in \mathcal{O}$, for $k=1, \ldots, n$. Then $J=d \mathcal{M}$ is an ideal of $\mathcal{O}$ [10, section 4.7]; the normalized generators $d \nu_{k}, k=1, \ldots, n$, are algebraic integers which form a $\mathbb{Z}$-basis for $J$. By construction, $\mathcal{M}$ is also a module over $\mathcal{O}$; furthermore $\mathcal{O}$ is the largest subring of $K$ with this property. Now $\sum_{i=1}^{N}\left|\Omega_{i}\right|=1 \in \mathcal{M}$, and so $\mathbb{Z} \subset \mathcal{M}$. We can form the quotient

$$
\mathcal{M}^{\prime}:=\mathcal{M} / \mathbb{Z}
$$

which is a $\mathbb{Z}$-module, which we shall represent as $\mathcal{M} \cap[0,1)$. Let $j$ be the smallest positive integer in $J$ : then the rational numbers in $\mathcal{M}$ are the multiples of $j / d$, and hence

$$
(\mathbb{Q} \cap \mathcal{M}) / \mathbb{Z}=\frac{j}{d} \mathbb{Z} / \mathbb{Z} \cong \mathbb{Z} / b \mathbb{Z} \quad b=\frac{d}{\operatorname{gcd}(d, j)},
$$

that is, there are $b$ congruence classes of rational numbers in $\mathcal{M}^{\prime}$, while the non-rational part is freely generated. Thus we have a $\mathbb{Z}$-module isomorphism $\mathcal{M}^{\prime} \rightarrow \mathbf{L}^{\prime}$ where

$$
\mathbf{L}^{\prime}=(\mathbb{Z} / b \mathbb{Z}) \oplus \mathbb{Z}^{n-1}
$$

\footnotetext{
${ }^{1}$ A finitely-generated subring of $K$, which contains 1 and has maximal rank.
} 
which characterizes the additive structure associated with the map E. Accordingly, we choose for $\zeta \in \mathcal{M}^{\prime}$ the following representation

$$
\zeta=\frac{1}{d} \sum_{k=1}^{n} m_{k} \nu_{k}^{\prime} \quad m_{k} \in \mathbb{Z} \quad \nu_{k}^{\prime} \in J, \quad \nu_{1}^{\prime}=j,
$$

where the $\nu_{k}^{\prime}$ are obtained from the normalized generators $d \nu_{k}$ via a unimodular transformation. The isomorphism (9) then becomes

$$
\phi^{\prime}: \zeta \mapsto\left(m_{0}(\bmod b), m_{1}, \ldots, m_{n-1}\right) .
$$

To represent the dynamics in the field $K$-the extension of the rational generated by the intervals' lengths - we use the $\mathbb{Z}$-basis $\left(\nu_{k}\right)$ for $\mathcal{M}$, and any point $x$ in $K$ can be written as

$$
x=\sum_{k=1}^{n} r_{k} \nu_{k} \quad \text { for } \quad r_{k} \in \mathbb{Q} .
$$

By definition, $E$ preserves $\mathcal{M}$ and for any $x \in K$ its orbit is confined in $x+\mathcal{M}$. We have a "layered" representation of $K$ and we regard it as the union of disjoint invariant sets of the form

$$
K=\bigcup_{\xi \in \Xi}(\xi+\mathcal{M})
$$

for a chosen set of representative $\Xi$. Following [18], we choose

$$
\Xi=\left\{\sum_{k=1}^{n} r_{k} \nu_{k}, \quad r_{k} \in \mathbb{Q} \cap[0,1)\right\} .
$$

The dynamics of $E$ acts on each layer separately, which in each case may be represented as the lattice $\mathbf{L}$, with a $\xi$-dependent dynamics on it.

In general, the closer two points get in $\mathcal{M}$, the further away their images in the lattice $\mathbf{L}$ will be. Indeed, let $x \in \mathcal{M}$ correspond to the lattice point $z=\left(z_{1}, \ldots, z_{n}\right)$

$$
x=\sum_{k=1}^{n} z_{k} \nu_{k} \in \mathcal{M}
$$

If the coefficients $z_{k}$ are bounded, then there are only finitely many possibilities for $x$, and hence $x$ is bounded away from zero. Thus if $|x| \rightarrow 0$ then $\|z\| \rightarrow \infty$, and one is interested in the relation between these limits.

We recall that height of a polynomial is the maximum absolute value of the coefficients, and the height of an algebraic number is the height of its minimal polynomial. In the particular case when $\mathcal{M}$ takes the form $\mathcal{M}=\mathbb{Z}[\lambda]$ for some algebraic number $\lambda$ of degree $n, 2$ Liouville's inequality gives the following bound.

\footnotetext{
${ }^{2} \mathbb{Z}[\lambda]$ is the $\mathbb{Z}$-module generated by $1, \lambda, \lambda^{2}, \ldots, \lambda^{n-1}$
} 
Proposition 2 If $\mathcal{M}=\mathbb{Z}[\lambda]$ for some algebraic number $\lambda$ of degree $n$, then for every $x \in \mathcal{M}$ we have

$$
|x|\|z\|^{c} \geq e^{c(n-1)} \quad z=\phi(x) \quad c=\operatorname{deg} \lambda+\log H(\lambda)
$$

where $H(\lambda)$ is the height of $\lambda$.

Proof: We have (cf. [24]) that for an integer polynomial $f$ with height $\leq H$ and degree $d$, for all algebraic number $\lambda$ we have either

$$
|f(\lambda)| \geq e^{-c(d+\log H)}
$$

or $\lambda$ is a root of $f$. The constant is $c=\operatorname{deg} \lambda+H(\lambda)$. In our context, the polynomial $f$ has coefficients $z_{i}$, and $\lambda$ is the generator of $\mathcal{M}$, that is, the basis vectors $\nu_{k}=\lambda^{k-1}$. Then $H=\|z\|$ for the max-norm and

$$
|f(\lambda)|=|x| \geq e^{-c(n-1)}|| z \|^{-c}
$$

as desired.

\subsection{Drift vector}

Let $v_{i}$ be the image on the lattice of the translation vector $\tau_{i}$-see equation (6). The $d r i f t$ vector $\mathcal{S}$, defined as

$$
\mathcal{S}=\sum_{i=1}^{N}\left|\Omega_{i}\right| v_{i}=\pi(\Lambda)
$$

gives the dominant direction of the dynamics over the lattice. The drift vector is related to a known invariant of IET theory, the so-called Sah-Arnoux-Fathi invariant, as we shall see in section 2.4.

Let the point $x \in \mathcal{M}$ have itinerary $\omega$, and let $z=\phi(x)$ be the corresponding lattice point. Then the orbit of $z$ may be expressed in terms of the drift vector as follows

$$
z+\pi s\left(\omega^{(k)}\right)=z+k \mathcal{S}+\pi D_{k}=z+k(\mathcal{S}+\alpha(k))
$$

where the staircase function $s$ and the discrepancies $D_{k}$ were defined in equations (3) and (5)), respectively, and $\alpha(k)=\pi D_{k} / k$. Because $\left|D_{k}\right|=o(k)$, the orbits of any lattice map with nonzero drift vector will be dominated by a linear drift in the direction of $\mathcal{S}$.

\subsection{Density}

The dynamics in $\mathbf{L}$ takes place on a subset of zero density. We can nonetheless have a meaningful measure of the "size" of the orbits by defining a modified density function on 
the reduced lattice $\mathbf{L}^{\prime}$, which results from restriction to the unit interval. With reference to (8) and (9), we represent a subset $A$ of $\mathcal{M}^{\prime}$ as

$$
A=A_{0}+\cdots+A_{b-1} \quad A_{i} \cap \sum_{k \neq i} A_{k}=\{0\} \quad A_{i} \cap \mathbb{Q} \equiv \frac{i}{b}(\bmod \mathbb{Z}) .
$$

Letting $\mathbf{A}_{j}=\phi\left(A_{j}\right)$, we define

$$
D\left(\mathbf{A}_{j}\right)=\lim _{k \rightarrow \infty} \frac{1}{b(2 k)^{n-1}} \sharp\left\{\left(m_{0}(\bmod b), m_{1}, \ldots, m_{n-1}\right) \in \mathbf{A}_{j},\left|m_{i}\right| \leq k, i \neq 0\right\} .
$$

Note that $m_{0}(\bmod b)$ is fixed, by construction. If the limit above exists for all $j$, we define the density of $\mathbf{A}$ as

$$
\mathcal{D}(\mathbf{A})=\sum_{j=0}^{b-1} \mathcal{D}\left(\mathbf{A}_{j}\right)
$$

which is a (non countably additive) probability measure on $\mathbf{L}^{\prime}$.

The density $\mathcal{D}$ is invariant under an unimodular transformation $U$. The density $\mathcal{D}$ measures the proportion of the points lying inside an hyper-cube $\mathcal{C}_{k}$ centred at the origin, with side $2 k$, as $k$ becomes large. Considering $\mathcal{D}(U \mathbf{A})$ is equivalent to consider $\mathcal{D}^{\prime}(\mathbf{A})$ where $\mathcal{D}^{\prime}$ measures the limiting proportion of the points lying inside the rhombus $U^{-1} \mathcal{C}_{k}$, also centred on the origin. As for all $k$ there exists $k^{\prime}$ and $k^{\prime \prime}$ such that $U^{-1} \mathcal{C}_{k^{\prime}} \subset \mathcal{C}_{k} \subset U^{-1} \mathcal{C}_{k^{\prime \prime}}$ we deduce that $\mathcal{D}(\mathbf{A})=\mathcal{D}^{\prime}(\mathbf{A})=\mathcal{D}(U \mathbf{A})$.

Proposition 3 Let $I \subset[0,1)$ be a finite union of intervals, and let $\mathbf{A}=\phi(\mathcal{M} \cap I)$. Then $\mathcal{D}(\mathbf{A})$ exists and is equal to the Lebesgue measure of $I$.

Proof. We begin with the case in which $I$ is a single interval. Let $\mathbf{m}=\left(m_{0}, \ldots, m_{n-1}\right)$, let $x_{\mathbf{m}}=\phi^{-1}(\mathbf{m})$, and let

$$
C_{k}=\frac{1}{b(2 k)^{n-1}} \sharp\left\{\mathbf{m} \in \mathbf{A}:\left|m_{i}\right| \leq k\right\} \quad k=1,2, \ldots
$$

Now let $m_{0}, m_{2}, \ldots, m_{n-1}$ be fixed integers, and define

$$
C_{m_{0}, k, m_{2}, \ldots, m_{n-1}}^{\prime}=\frac{1}{2 k} \sharp\left\{\left(m_{0}, m, m_{2}, \ldots, m_{n-1}\right):|m| \leq k, x_{m_{0}, m, m_{2}, \ldots, m_{n-1}} \in I\right\}
$$

then by Weyl's criterion

$$
C_{m_{0}, k, m_{2}, \ldots, m_{n-1}}-|I| \rightarrow 0 \quad \text { when } \quad k \rightarrow \infty .
$$

We then have

$$
C_{k}=\frac{1}{b(2 k)^{n-2}} \sum_{m_{0}=0}^{b-1} \sum_{m_{2}, \ldots, m_{n-1}=-k}^{k} C_{m_{0}, k, m_{2}, \ldots, m_{n-1}}^{\prime}
$$

and

$$
C_{k}=\frac{1}{b(2 k)^{n-2}} \sum_{m_{0}=0}^{b-1} \sum_{m_{2}, \ldots, m_{n-1}=-k}^{k}\left(\epsilon_{m_{0}, k, m_{2}, \ldots, m_{n-1}}+|I|\right)
$$


where $\epsilon_{m_{0}, k, m_{2}, \ldots, m_{n-1}}=C_{m_{0}, k, m_{2}, \ldots, m_{n-1}}-|I|$. Finally

$$
C_{k}=|I|+\frac{1}{b(2 k)^{n-2}} \sum_{m_{0}=0}^{b-1} \sum_{m_{2}, \ldots, m_{n-1}=-k}^{k} \epsilon_{m_{0}, k, m_{2}, \ldots, m_{n-1}}
$$

the last term is a $(n-2)$-dimensional Cesàro sum and thus converges to zero when $k \rightarrow \infty$, leading to $\mathcal{D}(\mathbf{A})=|I|$. The argument above is easily extended to the case of a finite union of intervals.

Next we have

Proposition 4 If the drift vector is non-zero and if $n>2$, then the density of any orbit on the lattice $\mathbf{L}^{\prime}$ is zero.

Proof. This is an easy consequence of formula (17). We have

$$
\left\|\psi^{k}(z)-z\right\|=\|\mathcal{S} k+k \alpha(k)\| \geq k|\|\mathcal{S}\|-\|\alpha(k)\|| \geq k \min _{k}|\|\mathcal{S}\|-\|\alpha(k)\|| .
$$

This latter minimum is attained because $1>\|\alpha(k)\| \rightarrow 0$. Thus the number of points inside the box centred at the origin and with side $2 k$ increases at most linearly with $k$. So, if the degree of the IET is greater than 2, then the lattice $\mathbf{L}^{\prime}$ has rank greater than one, leading to zero density.

In particular, an interval exchange transformation can have the finite decomposition property - each of its layers is the union of a finite number of orbits - only if it has zero drift. The actual value of the drift vector depends on the choice of the basis of $\mathcal{M}$; changing the basis is equivalent to apply a unimodular transformation on $\mathcal{S}$ which would indeed change its direction and magnitude. Nevertheless, we will see that the relevant property of $\mathcal{S}$ is mainly its nullity.

\subsection{The Sah-Arnoux-Fathi invariant}

In this section we regard $\mathbb{R}$ as a vector space over $\mathbb{Q}$, and consider the tensor product $\mathbb{R} \otimes \mathbb{R}$ of $\mathbb{Q}$-vector spaces. To a general $N$-interval exchange $E$ with set of length $\left|\Omega_{i}\right|$ and translation vectors $\tau_{i}$, Arnoux [5] associates an element $S$ of $\mathbb{R} \otimes \mathbb{R}$. The quantity $S$ can be computed by the formula

$$
S=\sum_{i=1}^{N}\left|\Omega_{i}\right| \otimes \tau_{i}
$$

The Sah-Arnoux-Fathi (SAF) invariant is a non-trivial conjugacy invariant; it is not a complete invariant but is in some sense the best possible one. The set of all the IETs constitute a group under the composition law. The map associating to each IET its invariant $S$ is a homomorphism from the group of IETs into $\mathbb{R} \otimes \mathbb{R}$, with the smallest possible kernel. For further details and discussions about the algebraic significance of $S$ 
see $[5,26]$. From these references we also know that $S$ is an induction invariant for minimal transformations, i.e., $S$ is the same computed on any induced maps of a given minimal interval exchange transformation. If $E$ is periodic, then its invariant is zero. The converse is true for an interval exchange of three sub-intervals, but not for more subintervals, as shown in [5]. The Arnoux-Yoccoz maps [4,7] provide an example of this phenomenon: they have 7 sub-intervals and zero invariant, but they are not periodic, in fact they are minimal and uniquely ergodic.

In general, $\mathbb{R} \otimes \mathbb{R}$ is infinite dimensional, but in our case the lengths and the translations belong to $K$, which is a vector space over $\mathbb{Q}$ of dimension $n$. In fact we can even restrict ourselves to the $\mathbb{Z}$-module $\mathcal{M}$. The quantity $S$ should then be in $\mathcal{M} \otimes_{\mathbb{Z}} \mathcal{M}$ (the subscript $\mathbb{Z}$ emphasizes that the tensorial product is taken between $\mathbb{Z}$-modules). As above, we fix a $\mathbb{Z}$-basis $\nu_{1}, \ldots, \nu_{n}$ for $\mathcal{M}$. We define a linear map $V: \mathcal{M} \otimes_{\mathbb{Z}} \mathcal{M} \rightarrow \mathcal{M}^{n}$ by specifying its action on this basis as follows

$$
V: \nu_{i} \otimes \nu_{j} \longmapsto \underbrace{\left(0, \ldots, \nu_{i}, \ldots, 0\right)}_{\nu_{i} \text { has position } j} .
$$

The map $V$ is bijective and is an isomorphism of $\mathbb{Z}$-modules. We have

$$
V(S)=\sum_{i=1}^{N} V\left(\left|\Omega_{i}\right| \otimes \tau_{i}\right) .
$$

Next we express the translation vectors $\tau_{i}$ and lengths $\left|\Omega_{i}\right|$ with respect to the module basis

$$
\tau_{i}=\sum_{k=1}^{n} \tau_{i}^{(k)} \nu_{k} \quad\left|\Omega_{i}\right|=\sum_{j=1}^{n}\left|\Omega_{i}\right|^{(j)} \nu_{j}
$$

so that

$$
\left|\Omega_{i}\right| \otimes \tau_{i}=\sum_{j, k=1}^{n}\left|\Omega_{i}\right|^{(j)} \tau_{i}^{(k)} \nu_{j} \otimes \nu_{k} .
$$

Finally, recalling that $v_{i}=\phi\left(\tau_{i}\right)$ are the images of the translation vector on $\mathbf{L}$ (equation (6)), we have

$$
\begin{aligned}
V(S) & =\sum_{i=1}^{N}\left(\tau_{i}^{(1)} \sum_{j=1}^{n}\left|\Omega_{i}\right|^{(j)} \nu_{j}, \ldots, \tau_{i}^{(n)} \sum_{j=1}^{n}\left|\Omega_{i}\right|^{(j)} \nu_{j}\right) \\
& =\sum_{i=1}^{N}\left(\tau_{i}^{(1)}, \ldots, \tau_{i}^{(n)}\right)\left|\Omega_{i}\right|=\sum_{i=1}^{N} v_{i}\left|\Omega_{i}\right|=\mathcal{S}
\end{aligned}
$$

which shows that the drift vector $\mathcal{S}$ is a representation of the SAF invariant in our specific context.

The condition $\mathcal{S}=0$ is quite strong; in fact it implies $n$ rational dependencies between the lengths of the intervals. Note that if we have only two intervals, the two lengths are of the form $\Omega_{1}$ and $1-\Omega_{1}$ and the condition $\mathcal{S}=0$ would imply $\left|\Omega_{1}\right| k+\left(1-\left|\Omega_{1}\right|\right) l=0$ for two integers $k$ and $l$, which is impossible since $\left|\Omega_{1}\right|$ is irrational. In fact a two-interval exchange is a pure rotation of the circle, which gives a pure translation on the lattice. 
More generally, for $N$ intervals, we have $N$ lengths $\left|\Omega_{i}\right| \in \mathcal{M}$, which we assumed to have rank $n$. For $\mathcal{S}$ to be zero, we must have

$$
\sum_{i=1}^{N} x_{i}\left|\Omega_{i}\right|=\sum_{i=1}^{N-1}\left(x_{i}-x_{N}\right)\left|\Omega_{i}\right|+x_{N}=0
$$

for some integers $x_{i}$. Then a necessary condition for $\mathcal{S}=0$ is $n<N-1$.

The following result summarizes the considerations above.

Proposition 5 The SAF invariant of an algebraic IET is zero if and only if its drift vector is zero. If the degree $n$ and the number of intervals $N$ are such that $n \geq N-1$, then the drift vector, and hence the SAF invariant, are non-zero.

By the discussion above, the Arnoux-Yoccoz [4,7] and Arnoux-Rauzy [6] families of IET have zero SAF invariant and thus zero drift vector.

\section{Renormalizable cases}

\subsection{Algebraic consequences of renormalizability}

In the following, we assume $E$ to be renormalizable. An IET of $N$ intervals is renormalizable if it has the same dynamics at each scale level, meaning that there exists an interval $I \subset[0,1)$ with the property that the induced map on $I$ is conjugated to the IET itself via a simple rescaling $\rho$. Thus the induced map on $I$ is an IET of $N$ intervals whose lengths are $\rho\left|\Omega_{i}\right|$ with a common scaling factor $\rho$. In this section, we prove the following theorem.

Theorem 6 Let $E$ be uniquely ergodic interval exchange transformation on $N$ intervals, which is renormalizable with scaling ratio $\rho$. Then $\rho$ is a unit of degree $\leq N$ in the ring $\mathbb{Z}[\beta]$, where $\beta=\rho^{-1}$ is the largest eigenvalue of the incidence matrix of the primitive substitution associated with $E$. Furthermore, $E$ is defined over $\mathbb{Q}(\rho)$ and it has maximal rank.

Proof. By construction, the scaled lengths can be expressed as integral linear combinations of the lengths $\left|\Omega_{i}\right|$. If we represent these linear combinations as an $N$ by $N$ integral matrix $B$, then we see that, by definition, the lengths vector $\Lambda$ is an eigenvector of $B$ with eigenvalue $\rho$. This tells us that $\rho$ is an algebraic integer, and if $n$ is its degree, then $n \leq N$. Since $B \cdot \Lambda=\rho \Lambda$ and $\Lambda$ is a probability vector, then

$$
\sum_{i=1}^{N}(B \cdot \Lambda)_{i}=\rho \sum_{i=1}^{N}\left|\Omega_{i}\right|=\rho .
$$


We see that $\rho \mathcal{M} \subset \mathcal{M}$ and $\rho \in \mathcal{M}$. Now, $\mathcal{M}$ contains 1 , and repeated scaling show that it also contains all positive powers of $\rho$. Hence $\mathcal{M}$ contains the ring $\mathbb{Z}[\rho]$. It follows that the rank of $\mathcal{M}$ is at least as large as the degree $n$ of $\rho$.

A renormalizable and uniquely ergodic interval exchange is conjugated to a primitive substitution $\sigma$. Furthermore, the atoms of the induced map on $I$ have their iterates cover the whole interval $\Omega$, lest there would be either some periodic cells or some different ergodic component. We conclude that

$$
\sum_{i=1}^{N} \rho^{k}\left|\sigma^{k}(i)\right|\left|\Omega_{i}\right|=1 \quad k \geq 0
$$

Because the substitution is primitive, for all $i$ the quantity $\left|\sigma^{k}(i)\right|$ grows like $\beta^{k}$, the powers of the Perron eigenvalue of the incidence matrix $M_{\sigma}$ of $\sigma$, and we have

$$
\rho=\beta^{-1} \text {. }
$$

Because both $\rho$ and $\beta$ are algebraic integers, they are units.

Next we show that $\Lambda$ is the positive eigenvector of $M_{\sigma}$ associated with $\beta$. Considering the fixed point $\omega$ of $\sigma$, let $j$ be the unique integer with the property that $\sigma^{k}(j)$ is an approximation to $\omega$. We find

$$
M_{\sigma}\left(\begin{array}{c}
\left|\Omega_{1}\right| \\
\vdots \\
\left|\Omega_{N}\right|
\end{array}\right)=\lim _{k \rightarrow \infty} \frac{1}{\left|\sigma^{k}(j)\right|} M_{\sigma}\left(\begin{array}{c}
\left|\sigma^{k}(j)\right|_{1} \\
\vdots \\
\left|\sigma^{k}(j)\right|_{N}
\end{array}\right)=\lim _{k \rightarrow \infty} \frac{1}{\left|\sigma^{k}(j)\right|}\left(\begin{array}{c}
\left|\sigma^{k+1}(j)\right|_{1} \\
\vdots \\
\left|\sigma^{k+1}(j)\right|_{N}
\end{array}\right)
$$

For all $i$, we have

$$
\frac{1}{\left|\sigma^{k}(j)\right|}\left|\sigma^{k+1}(j)\right|_{i}=\frac{\left|\sigma^{k+1}(j)\right|}{\left|\sigma^{k}(j)\right|} \times \frac{\left|\sigma^{k+1}(j)\right|_{i}}{\left|\sigma^{k+1}(j)\right|} \rightarrow \beta\left|\Omega_{i}\right| .
$$

Since $M_{\sigma} \Lambda=\beta \Lambda$, for the same reason as above we have $\beta \in \mathcal{M}$, and as $\beta \mathcal{M} \subset \mathcal{M}$ and $\beta^{-1} \mathcal{M}=\rho \mathcal{M} \subset \mathcal{M}$, we have $\beta \mathcal{M}=\rho \mathcal{M}=\mathcal{M}$. We see that the vector of the lengths is the solution of the system of equations

$$
M_{\sigma} z=\beta z \quad \text { and } \quad \sum_{i=1}^{N} z_{i}=1 \quad z=\left(z_{1}, \ldots, z_{N}\right) .
$$

This solution is unique. Indeed, by the Perron-Frobenius theorem, $\operatorname{dim} \operatorname{ker}\left(M_{\sigma}-\beta \mathbf{1}\right)=1$, and the solution belongs to $\mathbb{Q}(\beta)=\mathbb{Q}(\rho)$. Then $\mathcal{M} \subset \mathbb{Q}(\rho)$, and therefore the rank of $\mathcal{M}$ is at most $n$. But then the rank is exactly $n$, namely, it's maximal.

The proof above shows that $\mathcal{M}$ is a $\mathbb{Z}[\rho]$-module, and hence $\mathbb{Z}[\rho]$ is a subring of the order $\mathcal{O}$ - see equation (7) and following remarks.

Let $R$ be the matrix representing the action of multiplication by $\rho$ on the lattice $\mathbf{L}$. Since $\rho$ is a unit, the matrix $R$ is unimodular; moreover, from linear algebra and the 
fact that $\rho$ has degree $n$, we have that the characteristic polynomial of $R$ is equal to the minimal polynomial of $\rho$. In particular, $\rho$ is an eigenvalue of $R$.

The codes of the orbits from a scale to the next can be found by mean of the substitution $\sigma$. If a point $x$ belongs to the atom $i$, we have

$$
E^{|\sigma(i)|}(\rho x)=\rho E(x),
$$

or on the lattice

$$
\psi^{|\sigma(i)|}(R z)=R \psi(z) .
$$

Letting the projection $\pi$ be as in (6), we have

$$
\begin{aligned}
\psi^{|\sigma(i)|}(R z) & =R z+\sum_{j=1}^{|\sigma(i)|} v_{\sigma(i)_{j}}=R z+\sum_{j=1}^{N}|\sigma(i)|_{j} v_{j}=R z+\pi \sum_{j=1}^{N}|\sigma(i)|_{j} e_{j} \\
& =R z+\pi M_{\sigma} \sum_{j=1}^{N}|i|_{j} e_{j}=R z+\pi M_{\sigma} e_{i} .
\end{aligned}
$$

By equation (19), we then have

$$
R \psi(z)=R z+R v_{i}=R z+\pi M_{\sigma} e_{i}
$$

and since $\pi e_{i}=v_{i}$

$$
R \pi e_{i}=\pi M_{\sigma} e_{i} \quad i=1, \ldots, N
$$

Finally

Lemma 7 The following diagram commutes

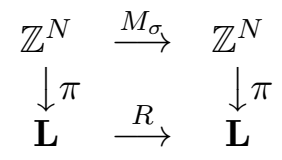

As a corollary, we see that if $X$ is an eigenvector of $M_{\sigma}$ with eigenvalue $\zeta$ and $X$ is not in the kernel of $\pi$, then $\pi X$ is an eigenvector of $R$ with the same eigenvalue

$$
\pi M_{\sigma} X=\zeta \pi X=R \pi X
$$

In addition, the matrix $M_{\sigma}$ preserves $\operatorname{ker} \pi$. Indeed if $X \in \operatorname{ker} \pi$, then again $R \pi X=0=$ $\pi M_{\sigma} X$ and $M_{\sigma} X \in \operatorname{ker} \pi$. In particular, the characteristic polynomial of $M_{\sigma}$ has a factor of degree $N-n$.

We already know that $\Lambda$ is an eigenvector of $M_{\sigma}$ with the Perron eigenvalue $\beta$. We thus have two possibilities: i) $\pi \Lambda \neq 0$ (the drift vector is non-zero) and $\pi \Lambda$ is an eigenvector of $R$ with eigenvalue $\beta$; ii) $\Lambda \in \operatorname{ker} \pi$ and $\beta$ is not part of the spectrum of $R$. This is because the spectrum of $R$ is contained in the spectrum of $M_{\sigma}$ and the Perron eigenvalue is simple. 


\subsection{Connections with drift vector}

When the drift vector $\mathcal{S}$ is non-zero, the algebraic number $\beta$ is in the spectrum of $R$. This means that $\rho$ and $\beta=\rho^{-1}$ are algebraic conjugates, namely roots of the same irreducible polynomial $p(x)$ of degree $n$. In fact, all eigenvalues of $R$ are algebraic conjugates and all are units.

Let now $\tilde{p}(x)=x^{\operatorname{deg} p} p(1 / x)$ be the reciprocal polynomial of $p$ - the polynomial whose roots are the reciprocal of the roots of $p$. Because $p$ is irreducible, so is $\tilde{p}$, and since $\beta$ is a root of both $p$ and $\tilde{p}$, it follows that $p=\tilde{p}$. So the roots of $p$ regroup into reciprocal pairs, and since these roots cannot include \pm 1 , the degree $n$ of $p$ must be even. Any self-reciprocal polynomial $p$ of even degree takes the form (see for instance [2])

$$
p(x)=x^{n / 2} h\left(x+x^{-1}\right)
$$

for some an integral polynomial $h$ of degree $n / 2$. The polynomial $h$ is irreducible because $p$ is. So if $\zeta$ is root of $p$, then $\xi=\zeta+\zeta^{-1}$ is root of $h$, and since $\zeta^{2}-\xi \zeta+1=0$, we see that $\zeta$ lies in a quadratic extension of the algebraic number field generated by $\xi$.

Now $p$ is the characteristic polynomial of both $R$ and $R^{-1}$, which have the same spectrum. Because $p$ is a minimal polynomial, its roots all have multiplicity one and therefore $R$ and $R^{-1}$ are diagonalizable (see, for instance, [11, $\left.\S 3.10\right]$ ). It then follows that $R$ and $R^{-1}$ are conjugate, indeed integrally conjugate. That is, there exists an integral matrix $G$ such that

$$
R^{-1}=G R G^{-1}
$$

showing that $R$ has time-reversal symmetry [15]. Then $G$ is an involution, exchanging two subspaces of dimension $n / 2$.

When the drift vector $\mathcal{S}$ is zero, the eigenvalue $\beta$ of $M_{\sigma}$ is not in the spectrum of $R$. Then the characteristic polynomial $P(x)$ of $M_{\sigma}$ admits the factorization

$$
P(x)=p(x) \tilde{p}(x) q(x)
$$

where the polynomials $p$ and $\tilde{p}$ are irreducible of degree $n$, while $q$ is a polynomial of degree $N-2 n$.

Summing up the results of this section we have

Proposition 8 Consider a uniquely ergodic algebraic IET on $N$ intervals, degree $n$ and maximal rank.

i) If the drift vector is non-zero and $n$ is odd, then renormalization is not possible;

ii) if the drift vector is zero then $2 n<N$. 
Note that if we have only two intervals, the two lengths are of the form $\Omega_{1}$ and $1-\Omega_{1}$ and the condition $S=0$ would imply $\left|\Omega_{1}\right| k+\left(1-\left|\Omega_{1}\right|\right) l=0$ for two integers $k$ and $l$ which is impossible as $\left|\Omega_{1}\right|$ is irrational. In the case when $n=N=2$, the drift vector must be non-zero and yet the map is known to be renormalizable, from the Boshernitzan and Carroll theorem [9].

\subsection{Recursive tiling property}

We already introduced the standard coding, with respect to the partition of the interval $\Omega$. In $[14,16,18]$ and elsewhere, a different type of coding is also used, which is based on the so-called recursive tiling property. As we mentioned earlier (in the proof of theorem 6), for each $k$, the interval $\Omega$ splits into $\sum_{i=1}^{N} \sigma^{k}(i)$ sub-intervals, each corresponding to a $k$-cylinder in the symbolic space. For a given point $x$, knowing for each $k$ in which cylinder $x$ lies is the essence of this new coding, which, for reasons explained later, is also called the Vershik coding.

We consider the substitution $\sigma$ introduced above, using the notation $\omega^{i}=\sigma(i)$. A prefix $\mu$ of a word $\omega_{1} \cdots \omega_{k}$ is a sub-word of the type $\omega_{1} \cdots \omega_{j}$, with $j<k$. Let $\mathcal{P}$ be the set of all prefixes of the words $\omega^{1}, \ldots, \omega^{N}$, together with the $N$ additional symbols $\epsilon_{1}, \ldots, \epsilon_{N}$, which will be regarded as prefixes of the 1 -symbol words $\omega_{1}^{1}, \ldots, \omega_{1}^{N}$, respectively. We use the notation $\mu^{i} \in \mathcal{P}$ to characterize an arbitrary prefix of $\omega^{i}$. Associated to this notation there is the function $\chi$ which extracts the "exponent" of a prefix, meaning that $\chi(\mu)=i$ if $\mu$ is a prefix of $\omega^{i}$. Finally, given a prefix $\mu$ of $\omega$, we denote by $\mu_{+}$the symbol immediately following $\mu$ in $\omega$. This quantity is well-defined, since $\mu$ a proper sub-word of $\omega$. hence

If we let $E_{\omega_{1}^{i} \ldots \omega_{p}^{i}} x=x+\tau_{\omega_{1}^{i}}+\ldots+\tau_{\omega_{p}^{i}}$, then for $\mu^{i} \in \mathcal{P}$ we have $E_{\mu^{i}} \rho \Omega_{i} \subset \Omega_{\mu_{+}^{i}}$, and

$$
\bigcup_{\substack{\mu \in \mathcal{P} \\ \mu_{+}=i}} E_{\mu} \rho \Omega_{\chi(\mu)} \subset \Omega_{i}
$$

and

$$
\Omega=\bigcup_{i=1}^{N} \bigcup_{\substack{\mu \in \mathcal{P} \\ \mu_{+}=i}} E_{\mu} \rho \Omega_{\chi(\mu)} .
$$

The same decomposition can be made on each scaled sub-intervals and we thus have a recursive tiling of $\Omega$. A given point $x \in \Omega$ can thus be located if we know for each $k$ in which scaled sub-interval it lies. We can show that there exists a sequence of prefixes $\left(\mu_{1}, \mu_{2}, \ldots\right) \in \mathcal{P}$ such that

$$
x \in \bigcap_{k=1}^{\infty} E_{\mu_{1}} \rho \ldots E_{\mu_{k}} \rho \Omega_{\chi\left(\mu_{k}\right)} .
$$

There is a consistency constraint on the sequence, namely

$$
\chi\left(\mu_{k}\right)=\left(\mu_{k+1}\right)_{+} .
$$

On the possible sequences in $\mathcal{P}^{\mathbb{N}}$, the dynamics of $E$ translates into an odometer-like process which can be conveniently represented as a Vershik map, thus justifying the terminology of "Vershik code". 
In [18], the periodic sequences of the Vershik code played an important role, firstly because it is possible to compute the associated points explicitly, and secondly because all the points of the lattice turned out to have periodic or eventually periodic Vershik codes. By construction, a point $x$ with periodic Vershik code $\left(\mu_{1} \ldots \mu_{T}\right)^{\infty}$ is an invariant point of a contraction

$$
E_{\mu_{1}} \rho \ldots E_{\mu_{T}} \rho x=x \quad \text { or } \quad x=\frac{1}{1-\rho^{T}} \sum_{i=0}^{T-1} \tau_{\mu_{i+1}} \rho^{i}
$$

where $\tau_{\mu_{i}}$ are the translations corresponding to the maps $E_{\mu_{i}}$, which belong to $\mathcal{M}$. The rightmost expression in (22) may be regarded as an expansion of $x$ in the base $\rho$ where the "digits" $\tau_{\mu_{i}}$ are algebraic numbers (this viewpoint is developed in [19]). Because the factors $1-\rho^{T}$ at denominator are not units, in general a periodic point is not an algebraic integer, but rather a point of the field $K$. By the decomposition (12), there exists a representative $\xi \in \Xi$ such that $x$ has its orbit on $\xi+\mathcal{M}$. We note that the set $\xi+\mathcal{M}$ is not invariant under scaling by $\rho$, that is,

$$
\rho \xi \in \xi^{\prime}+\mathcal{M} \quad \text { where } \quad \xi^{\prime} \equiv \rho \xi(\bmod \mathcal{M}) .
$$

Following [18], we notice that, for any positive integer $m$, the lattice $\mathcal{M} / m$ is an $\mathcal{O}$-module (see remarks following equation (7)), and in particular is invariant under multiplication by $\rho$. (Alternatively, note that multiplication by $\rho$ is represented as an integer matrix $R$ which preserves the lattices $\mathbf{L} / m$.) With our definition of $\Xi$, we then see that $\Xi \cap \mathcal{M} / m$ has $m^{3}$ elements, which limits the number of possible $\xi^{\prime}$ after multiplication. Thus, there exists an integer $t$ such that $\rho^{t} \xi \equiv \xi(\bmod \mathcal{M})$, and the smallest such $t$ is called the order of $\xi$. From equations (22), if the point $x=\beta+\xi$ with $\beta \in \mathcal{M}$ and $\xi \in \Xi$ has period $T$, we find that $\xi-\rho^{T} \xi \in \mathcal{M}$, which implies that $T$ must be a multiple of the order of $\xi$.

The period $T$ determines the maximum denominator of the fractional component $\xi$ of a periodic point, which is given by the determinant of $I-R^{T}$. Indeed, it is easy to see that the characteristic polynomial of $I-R^{T}$ is $p_{T}(1-t)$ where $p_{T}$ is the characteristic polynomial of $R^{T}$. Then the eigenvalues of this matrix are of the form $1-\zeta_{i}^{T}$, where $\zeta_{i}$ runs through the eigenvalue of $R$. From the previous section we know that we the eigenvalues of $R$ come in reciprocal pairs $(\zeta, 1 / \zeta)$, and a straightforward computation shows that

$$
d_{T}=\left|\operatorname{det}\left(I-R^{T}\right)\right|=\prod_{i=1}^{n / 2} \frac{\left(\zeta_{i}^{T}-1\right)^{2}}{\zeta_{i}^{T}}
$$

where the product is taken over all the $n / 2$ eigenvalues greater than one. There exists then a value of the period above which $d_{T}$ increases monotonically with $T$.

Next we prove a bound for points with eventually periodic code.

Proposition 9 Let $x$ be a point whose Vershik code is eventually periodic with transient $t$ and period $T$, and let $z=\phi(x)$. Then there exists a positive constant $C$ such that

$$
\|z\| \leq C\|R\|^{t+T}
$$

where $\|R\|$ is the induced norm of matrices. 
Proof: On the corresponding lattice, we have

$$
z=\frac{1}{I-R^{T}} \sum_{j=0}^{T-1} R^{j} v_{\mu_{j+1}} \quad v_{\mu_{j}}=\phi\left(\tau_{\mu_{j}}\right)
$$

and we see that

$$
\|z\| \leq\left\|\left(I-R^{T}\right)^{-1}\right\| \sum_{j=0}^{T-1}\left\|R^{j} v_{\mu_{j+1}}\right\| \leq\left\|\left(I-R^{T}\right)^{-1}\right\| \max _{k}\left\{\left\|v_{\mu_{k}}\right\|\right\} \sum_{j=0}^{T-1}\|R\|^{j},
$$

and thus

$$
\|z\| \leq C\left\|\left(I-R^{T}\right)^{-1}\right\| \frac{\|R\|^{T}-1}{\|R\|-1} .
$$

By the discussion above on the eigenvalues of $\left(I-R^{T}\right)$, we see that

$$
\left\|\left(I-R^{T}\right)^{-1}\right\|=\max _{k}\left|\frac{1}{\zeta_{k}^{T}-1}\right|=\max _{k}\left|\frac{1}{\zeta_{k}^{-T}-1}\right|=\max _{k}\left|\frac{\zeta_{k}^{T}}{1-\zeta_{k}^{T}}\right| .
$$

Now the expression in the max is very small if $\zeta_{k}<1$ and close to one if $\zeta_{k}>1$, it is clear that the maximum is attained for the maximum value of $\left|\zeta_{k}\right|$ (at least for sufficiently large $T)$, which happens to be $\|R\|$ and

$$
\left\|\left(I-R^{T}\right)^{-1}\right\|=\max _{k}\left|\frac{\zeta_{k}^{T}}{1-\zeta_{k}^{T}}\right|=\frac{\|R\|^{T}}{\|R\|^{T}-1}=1+\frac{1}{\|R\|^{T}-1} .
$$

We finally have

$$
\|z\| \leq\left(\frac{\max _{k}\left\{\| v_{\mu_{k}}||\right\}}{\|R\|-1}\right)\left(\|R\|^{T}-1\right) \leq C\|R\|^{T} .
$$

Let us now assume that the point $z$ has eventually periodic Vershik code $\left(\mu_{1} \ldots \mu_{t}\left(\mu_{t+1} \ldots \mu_{t+T}\right)^{\infty}\right)$ with transient length $t$ and period $T$. For each positive integer $t$, we define the mapping

$$
\nabla: \mathbf{L} \rightarrow \mathbf{L} \quad z \mapsto \psi_{\mu_{1}} R \cdots \psi_{\mu_{t}} R z=R^{t} z+\sum_{j=0}^{t-1} R^{j} v_{\mu_{j+1}}
$$

Then $\nabla_{t}^{-1} x$ has $T$-periodic Vershik code, and from the above we see that

$$
\|z\| \leq C|| \nabla_{t}|||| R\left\|^{T} \leq C\right\| R \|^{T}\left(\left.\|R\|\right|^{t}+\sum_{j=0}^{t-1}\|R\|\left\|^{t}\right\| v_{\mu_{j+1}} \|\right) .
$$

Applying again the geometric sum formula we see that we have a positive constant $C^{\prime}$ such that

$$
\|z\| \leq C C^{\prime}\|R\|^{T}\|R\|^{t}
$$

From this proposition, we know that the number of distinct points $z$ with prefix length $t$ and period $T$ which belong to a given layer $\xi+\mathcal{M}$ is of the order of $\|R\|^{(n-1)(t+T)}$. We also know that we have up to $d_{T}$ such layers compatible with this specific period $T$. 
The number of admissible prefixes of length $t$ is given by the admissibility matrix of the graph described by the relation (21). This graph can be described either as a Bratteli diagram or as a more conventional connected graph. In any way, it can be shown [21] that if $\sigma$ is a primitive substitution, then the admissibility matrix is primitive as well; moreover the maximum eigenvalue of this matrix is equal to the maximum eigenvalue of $M_{\sigma}$, the incidence matrix of the substitution. The number of admissible paths in the aforementioned graph, as well as all the number of $T$-cycles, are then of the order of powers of $\beta=\left\|M_{\sigma}\right\|$.

We now fix a period $T$, and consider all the prefix-lengths $t$. We see that in order that all the points fit on all the possible lattices, we must have

$$
d_{T}\|R\|^{(n-1)(t+T)} \geq C\left\|M_{\sigma}\right\|^{t+T}
$$

for some constant $C$. As $t$ increases, this is only possible if $\|R\|^{n-1} \geq\left\|M_{\sigma}\right\|$.

We have the following result.

\section{Proposition 10 If}

$$
\|R\|^{n-1}=\left\|M_{\sigma}\right\|,
$$

then for every period $T$ there is at least one layer $\mathcal{M}+\xi$ with the property that the set of (Vershik) eventually periodic points of period $T$ on the corresponding lattice has positive density.

Proof: The proof follows from the discussion above. Here we mention that the map sending each Bratteli code to a point in $\Omega$ is not one-to-one; however, it can be shown [21] that it is finitely many to one and thus it does not change the result.

If $\mathcal{S} \neq 0$ and $n>2$, then the identity (23) is never verified. Indeed, in this case, both matrices share the same maximum eigenvalue $\beta$, see comments at the end of section 3.1 . This is consistent with the remark we made above, that an orbit must have zero density in these cases.

In the case studied in [18], we had

$$
\|R\|^{2}=\left\|M_{\sigma}\right\|,
$$

though the result stated here is much less powerful that the results of [18].

\subsection{Remarks on asymptotic behaviour}

We begin by considering the orbit of the point $z$ which corresponds to the fixed point of the substitution. We know that there is an integer $j$ such that the code of $z$ begins with 
$\sigma^{k}(j)$ for all $k$. In this case, we have

$$
\begin{aligned}
\psi^{\left|\sigma^{k}(j)\right|}(z) & =z+\sum_{i=1}^{N}\left|\sigma^{k}(j)\right|_{i} v_{i} \\
& =z+\pi \sum_{i=1}^{N}\left\langle M_{\sigma}^{k} e_{j}, e_{i}\right\rangle e_{i} \\
& =z+\pi M_{\sigma}^{k} e_{j} \\
& =z+R^{k} v_{j} .
\end{aligned}
$$

We clearly see that the behaviour here is determined by the largest eigenvalue of $R$. If $\mathcal{S} \neq 0$, we know that this eigenvalue is $\beta$, and since $\left|\sigma^{k}(j)\right|$ also scales like $\beta^{k}$, we have a dominant linear behaviour, as expected.

If $\mathcal{S}=0$, then the behaviour is dominated by the largest eigenvalue of $M_{\sigma}$ which is also part of the spectrum of $R$. We have

$$
\pi D_{\left|\sigma^{k}(j)\right|}=R^{k} v_{j}=\pi M_{\sigma}^{k} e_{j}
$$

where the vector $D$ of discrepancies was defined in (5). The escape rate of the point is given by the discrepancy-vector of the point $z$. There are known results about the discrepancy of substitution dynamical systems, including a theorem from Adamczewski [1] which gives the growth rate of the maximum component of $D_{k}$, depending on the properties of the spectrum of $M_{\sigma}$. In particular, we have an upper bound of the form $(\log k)^{a} k^{b}$, with $a$ a positive integer and $0<b<1$. We have this growth when the second largest eigenvalue $\beta_{2}$ of $M_{\sigma}$ is greater than 1 (in modulus). The quantity $b$ has the form

$$
b=\frac{\log \left|\beta_{2}\right|}{\log \beta}
$$

and $a+1$ is the multiplicity of $\beta_{2}$

This indicates that a power-law behaviour can only occur when $\beta_{2}$ is greater than 1 in absolute value and has multiplicity one. This eigenvalue then dominates the evolution. Indeed if we have ||$\pi D_{\left|\sigma^{k}(j)\right|}|| \sim\left|\beta_{2}\right|^{k}$, since $\left|\sigma^{k}(j)\right| \sim \beta^{k}$ we are led to

$$
\left\|\pi D_{k}\right\| \sim k^{\frac{\log \left|\beta_{2}\right|}{\log \beta}} .
$$

In the case studied in [18], direct computation gives

$$
\frac{\log \left|\beta_{2}\right|}{\log \beta}=\frac{1}{2}
$$

which leads to positive density orbits on the lattice.

In general case the computation is less straightforward, but thanks to the recursive tiling property we can still derive estimates for the asymptotic behaviour. Let us thus consider a point $x$ having Vershik code $\left(\mu_{1} \ldots \mu_{k} \ldots\right)$. Then

$$
x \in E_{\mu_{1}} \rho \Omega_{\chi\left(\mu_{1}\right)} \cap E_{\mu_{1}} \rho E_{\mu_{2}} \rho \Omega_{\chi\left(\mu_{2}\right)} \cap \ldots
$$

and we can deduce that (see [21] for details)

$$
\begin{array}{ll}
x \in E_{\mu_{1}} \rho \Omega_{\chi\left(\mu_{1}\right)} & \Rightarrow \omega(x)=s^{\left|\mu_{1}\right|} \sigma\left(\chi\left(\mu_{1}\right)\right) \\
x \in E_{\mu_{1}} \rho E_{\mu_{2}} \rho \Omega_{\chi\left(\mu_{2}\right)} & \Rightarrow \omega(x)=s^{\left|\mu_{1}\right|} \sigma\left(s^{\left|\mu_{2}\right|} \sigma\left(\chi\left(\mu_{2}\right)\right)\right),
\end{array}
$$


where here $s$ denotes the shift map. To estimate the escape rate of the lattice point corresponding to $x$, we need to know the asymptotic number of occurrences of each letter in the words above. Let us call $L^{k}$ the vectors of the number of occurrences of each of the letters $1, \ldots, N$ in the $k$-th word above

$$
L_{i}^{k}=\left|s^{\left|\mu_{1}\right|} \sigma\left(\cdots s^{\left|\mu_{k}\right|} \sigma\left(\chi\left(\mu_{k}\right)\right) \cdots\right)\right|_{i} .
$$

The number of points in the beginning of the orbit of $x$ thus described is given by the length of the word above; since $L^{k}$ is a positive vector, this is given by

$$
\sum_{i=1}^{N} L_{i}^{k}=\left\|L^{k}\right\|
$$

Each shift cuts the number of every letter by a finite amount: the number of possible shift lengths is equal to the number of possible prefixes, and each one decreases each component of $L^{k}$ in a finite numbers of ways. If we call $\eta$ the maximum length of a prefix, then there exists not more than $N^{\eta}$ positive $N$-vectors $\varepsilon_{j}$ of norm not exceeding $N$ such that

$$
\begin{aligned}
L^{1} & =M_{\sigma} e_{\chi\left(\mu_{1}\right)}-\varepsilon_{\alpha_{1}} \\
L^{2} & =M_{\sigma}\left(M_{\sigma} e_{\chi\left(\mu_{2}\right)}-\varepsilon_{\alpha_{2}}\right)-\varepsilon_{\alpha_{1}} \\
& \vdots \\
L^{k} & =M_{\sigma}^{k} e_{\chi\left(\mu_{k}\right)}-\sum_{j=0}^{k-1} M^{j} \varepsilon_{\alpha_{j+1}},
\end{aligned}
$$

where $1 \leq \alpha_{i} \leq N^{\eta}$. As $k$ grows, the escape rate of $z$ under $\psi$ is thus given by the asymptotic behaviour of

$$
\sum_{i=1}^{N} L_{i}^{k} v_{i}=\pi L^{k}=R^{k} v_{\chi\left(\mu_{k}\right)}-\sum_{j=0}^{k-1} R^{j} \pi \varepsilon_{\alpha_{j+1}} .
$$

Since all integer vectors involved have uniformly bounded norm, we have the upper bound

$$
\left\|\psi^{\left\|L^{k}\right\|}(z)\right\|=\left\|\pi L^{k}\right\| \leq K \sum_{j=0}^{k}\|R\|^{j} \leq K \frac{\|R\| \|^{k+1}-1}{\|R\|-1} .
$$

This is not very sharp, yet there is no clear lower bound as one could adjust the $\varepsilon_{j}$ so as to make the $L^{k}$ bounded. The quantity $L^{k}$ is easier to express if $x$ has periodic or eventually periodic Vershik code.

In what follows, we state results only for periodic codes, to which an eventually periodic code can be reduced via a finite number of iterations of $E$. In the previous section, we saw that under suitable conditions, these lattice points constitute a "significantly large" set, thus justifying our interest in asymptotic estimates. We have the following result.

Proposition 11 If a point $x$ has periodic Vershik code of period $T$, then the corresponding lattice point $z=\phi(x)$ satisfies the asymptotic estimate

$$
\left\|\psi^{k T}(z)\right\| \sim k^{\frac{\log \|R\|}{\log \left\|M_{\sigma}\right\|}} \quad k \rightarrow \infty .
$$


Proof: Let $x$ have periodic Vershik code $\left(\mu_{1} \ldots \mu_{T}\right)^{\infty}$. By formula (22), every such point is representable as a lattice point. We define

$$
\delta_{T}=\sum_{j=0}^{T-1} R^{j} \pi \varepsilon_{\alpha_{j+1}}
$$

for some indices $\alpha_{j}$, and

$$
G(z)=R^{T} z+\delta_{T}
$$

The sequence

$$
\pi L^{k T}=G^{k}\left(v_{\chi\left(\mu_{k T}\right)}\right)
$$

is an arithmetic-geometric sequence with respect to $k$. We use the following lemma

Lemma 12 If $u_{k}$ is an arithmetic-geometric sequence, that is

$$
u_{k+1}=a u_{k}+b
$$

where $a$ is a square matrix such that $I-a$ is invertible and $b$ is a vector, then

$$
u_{k}=a^{k}\left(u_{0}-l\right)-l \quad \text { and } \quad \sum_{j=0}^{k} u_{j}=(I-a)^{-1}\left(I-a^{k+1}\right)\left(u_{0}-l\right)+k l
$$

with $l=(I-a)^{-1} b$.

Proof: We verify that the sequence

$$
v_{k}=u_{k}-l
$$

is a geometric sequence, indeed

$$
\begin{aligned}
v_{k+1} & =a u_{k}+b-(I-a)^{-1} b \\
& =a\left(u_{k}+a^{-1} b-a^{-1}(I-a)^{-1} b\right) \\
& =a\left(u_{k}+\left(a^{-1}(I-a)-a^{-1}\right)(I-a)^{-1} b\right) \\
& =a\left(u_{k}-(I-a)^{-1} b\right) .
\end{aligned}
$$

The result then comes by straightforward application of the classic formulae for geometric sequences.

We now apply the lemma to our case, with $a=R^{T}$ and $b=\delta_{T}$. Provided that $v_{\chi\left(\mu_{k T}\right)}=\left(I-R^{T}\right) \delta_{T}$, we obtain

$$
\left\|\psi^{\left\|L^{k T}\right\|}(z)\right\|=\left\|\pi L^{k T}\right\| \sim\|R\|^{k T}
$$

Furthermore, if we define

$$
\tilde{\delta}_{T}=\sum_{j=0}^{T-1} M_{\sigma}^{j} \varepsilon_{\alpha_{j+1}}
$$


with the corresponding indices $\alpha_{j}$ and if we call

$$
\tilde{G}(z)=M^{T} z+\tilde{\delta}_{T}
$$

then

$$
L^{k T}=\tilde{G}^{k}\left(e_{\chi\left(\mu_{k T}\right)}\right) .
$$

Applying again the argument above with $a=M_{\sigma}^{T}$ and $b=\tilde{\delta}_{T}$ yields

$$
\left\|L^{k T}\right\| \sim\left\|M_{\sigma}\right\|^{k T}
$$

which leads to

$$
\left\|\psi^{k T}(z)\right\| \sim k^{\frac{\log \|R\|}{\log \left\|M_{\sigma}\right\|}}
$$

This estimate is very similar to the one obtained for the orbit of zero, a point of constant Vershik code. Moreover, as the motion on the lattice is confined to a $(n-1)$-dimensional slab, if

$$
\frac{\log \|R\|}{\log \beta}=\frac{1}{n-1}
$$

we can expect positive density orbits.

\section{Examples}

In section 3.2 we saw that an algebraic IET of even degree and non-zero drift vector must fulfil certain conditions in order to be self-similar: the contraction ratio has to be a root of an irreducible self-reciprocal polynomial. A question then arises, naturally, is there any such IET? To answer this question, we shall use Rauzy-Veech induction, a powerful tool in IET theory, described in the pioneering papers of G. Rauzy and W. Veech $[23,25]$, and in many subsequent works.

Following [25], we let $E$ be an $N$-interval IET on the unit interval $\Omega$, with irreducible permutation $\pi$ and lengths vector $\Lambda$. We define

$$
I_{0}=\left[0, \sum_{i=1}^{N-1} \Lambda_{i}\right) \quad \text { and } \quad I_{1}=\left[0, \sum_{i=1}^{N-1} \Lambda_{\pi^{-1}(i)}\right) .
$$

Induction will be on the larger of these two intervals, $I$. Computing the first-return maps, one verifies that there are two cases:

- $\left|I_{0}\right|<\left|I_{1}\right|$ and the induced map has lengths vector $A_{0}(\pi)^{-1} \Lambda$ and permutation $a_{0} \pi$,

- $\left|I_{0}\right|>\left|I_{1}\right|$ and the induced map has lengths vector $A_{1}(\pi)^{-1} \Lambda$ and permutation $a_{1} \pi$. 
The permutations $a_{i} \pi$ and the matrices $A_{i}$ are defined as follows:

$$
a_{0} \pi j=\left\{\begin{array}{ll}
\pi j & \pi j \leq \pi N \\
\pi j+1 & \pi N \leq \pi j<N \\
\pi N+1 & \pi j=N
\end{array} \quad a_{1} \pi j= \begin{cases}\pi j & j \leq \pi^{-1} N \\
\pi N & j=\pi^{-1} N+1 \\
\pi(j-1) & \text { other } j\end{cases}\right.
$$

and

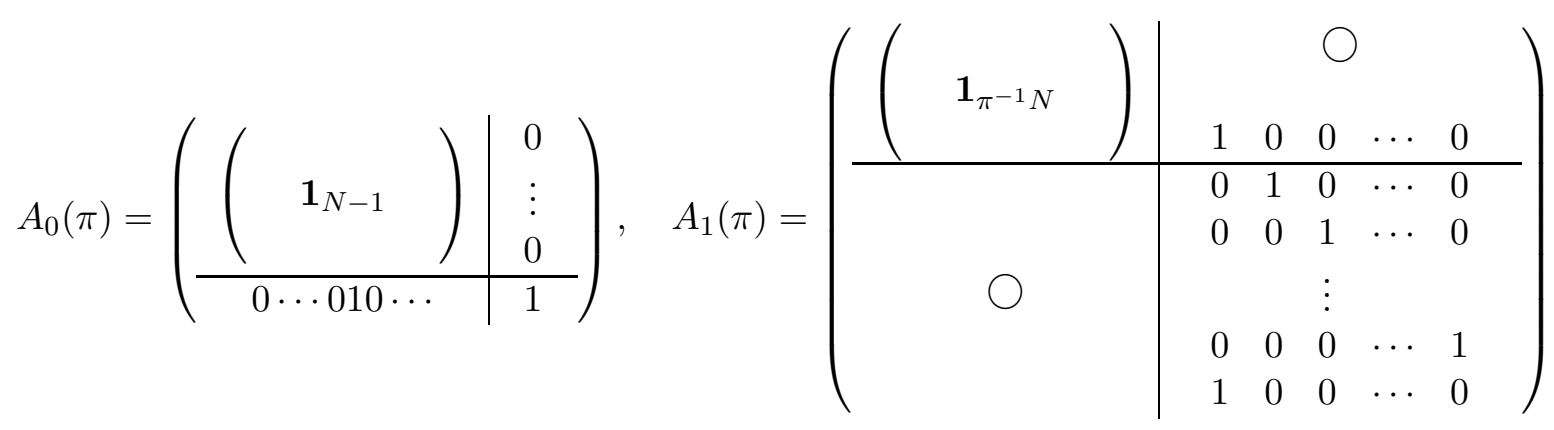

where here $\mathbf{1}_{n}$ denotes the $n$-dimensional identity matrix and the first 1 in the last line of $A_{0}(\pi)$ occurs at the $\pi^{-1} N$ position. Also, if $\pi^{-1} N=N-1$, the lower-right block of $A_{1}(\pi)$ contains the single element 1 .

The construction above is naturally associated with a Rauzy graph, whose vertices are the irreducible permutations on $N$ symbols. Each vertex has two outgoing edges, labelled 0 and 1, ending at the vertices labelled by the permutations of the induced IETs on $I_{0}$ and $I_{1}$. Each connected component of a Rauzy graph corresponds to a Rauzy class of IETs. The Rauzy classes for 3- and 4-interval IETs are shown in figures 13 ,

An IET on $N$ intervals is said to satisfy the Keane property [13] if the orbits of its discontinuity points are infinite and distinct. Such an IET is both minimal and uniquely ergodic; its unique invariant probability measure is Lebesgue measure. If $E$ is an IET on $N$ intervals with irreducible permutation and satisfying the Keane property (this holds for almost all length vectors), then, according to a theorem of Rauzy [23], the induced map described above is also an IET on $N$ intervals with irreducible permutation and also satisfies the Keane property.

Rauzy induction provides us with an effective tool for constructing self-similar IETs with a prescribed number of intervals. Indeed, a self-similar IET corresponds to a loop in the Rauzy graph, with the lengths of the induced map given by a positive eigenvector of the $N$-dimensional matrix obtained by taking the product of the $A_{i}(\pi)^{-1}$ around the loop, and a contraction factor given by the associated eigenvalue.

\subsection{Non-zero drift vector}

For three intervals, the Rauzy diagram has one component and three vertices - see figure 1. From proposition $8 i$ ), the drift vector cannot be zero, for otherwise $n$ would have to be 1 . But then $n$ must be even, from proposition $8 i i)$; as we clearly have $n \leq 3$, we 


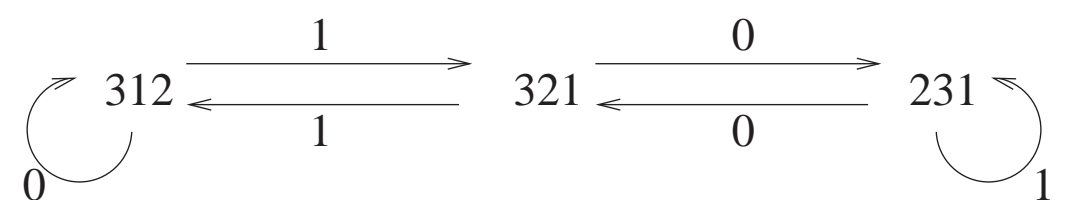

Figure 1: The Rauzy diagram for 3 intervals.

can only have quadratic cases. This means that the characteristic polynomial $p$ cannot be irreducible.

For four intervals, there are two Rauzy classes, shown in figures 2 and 3 . To find selfsimilar IETs, we checked all possible cycles of length at most 16 in a given Rauzy class and performed the corresponding products of the matrices $A_{i}(\pi)$. To obtain degree- 4 maps, we kept only those cycles with primitive product matrices and irreducible characteristic polynomials. For such a cycle, initiated at a specific permutation, the lengths of the map are the components of the normalized Perron eigenvector of the output matrix.

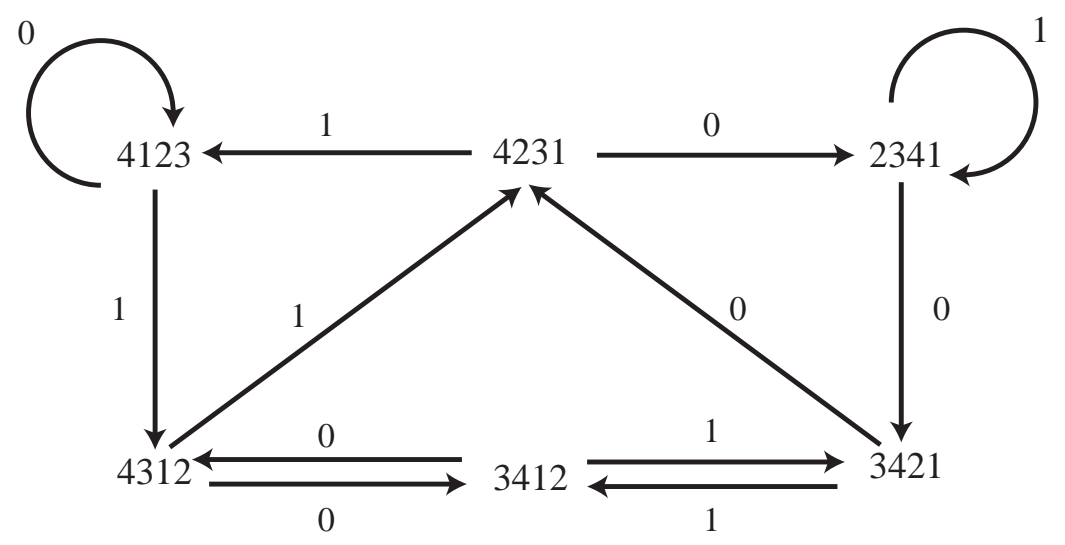

Figure 2: The first Rauzy class for 4 intervals.

Our first example of quartic IET over four intervals comes from the 8-cycle in the graph of figure 3, determined by the permutations $(4213,4213,4321,2431,3241,3241,4321,4132)$. As in section [3.1, we denote by $B$ the product matrix of this cycle

$$
B=\left(\begin{array}{llll}
1 & 1 & 1 & 1 \\
0 & 2 & 1 & 0 \\
1 & 2 & 2 & 1 \\
1 & 1 & 1 & 2
\end{array}\right)^{-1}
$$

with characteristic polynomial

$$
p(x)=x^{4}-7 x^{3}+13 x^{2}-7 x+1 .
$$

We see that $p(x)$ is indeed self-reciprocal and we verify that all his roots are real. The 


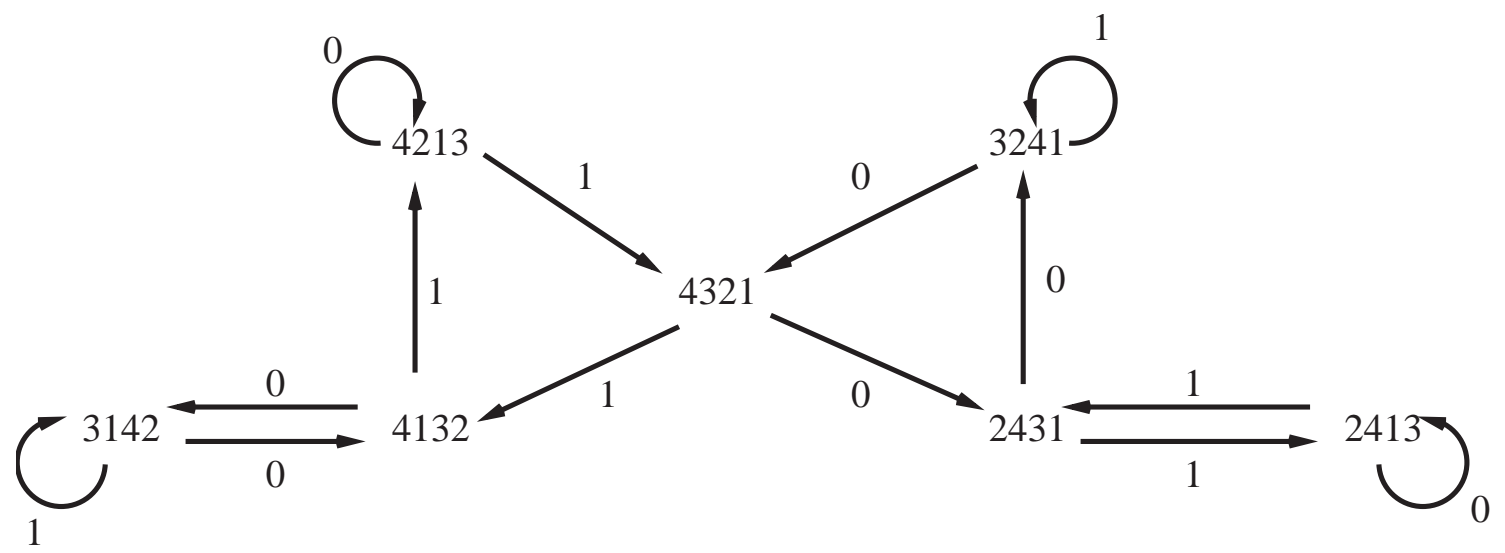

Figure 3: The second Rauzy class for 4 intervals.

smallest eigenvalue of $B$

$$
\rho=\frac{1}{4}(7+\sqrt{5}-\sqrt{38+14 \sqrt{5}}),
$$

corresponds to the normalized eigenvector

$$
\Lambda=\left(\rho, 1-4 \rho+\rho^{2}, 1-4 \rho+5 \rho^{2}-\rho^{3},-1+7 \rho-6 \rho^{2}+\rho^{3}\right)
$$

whose entries are positive and sum up to unity. These data, together with the permutation (4213) define an IET $E$, with module $\mathcal{M}=\mathbb{Z}[\rho]$, for which we choose the canonical basis $\nu_{i}=\rho^{i-1}, i=1, \ldots, 4$. One verifies that the induced map on the first interval $\Omega_{1}$ is just the original map $E$ rescaled by a factor $\rho$. Figure 4 shows the action of the map.

\begin{tabular}{|l|l|l|l|}
\hline 1 & 2 & 3 & 4 \\
\hline
\end{tabular}

Figure 4: The interval exchange transformation $E$

The following matrix transforms the lengths into the translations vector $\tau=\Delta \cdot \Lambda$ :

$$
\Delta=\left(\begin{array}{cccc}
0 & 1 & 1 & 1 \\
-1 & 0 & 1 & 0 \\
-1 & -1 & 0 & 0 \\
-1 & 0 & 0 & 0
\end{array}\right)
$$

The IET $E$ is conjugated to its first return in the interval $\Omega_{1}$ via the substitution

$$
\sigma:\left\{\begin{array}{lll}
1 & \longrightarrow & 143 \\
2 & \longrightarrow & 143223 \\
3 & \longrightarrow & 14323 \\
4 & \longrightarrow & 1443 .
\end{array}\right.
$$

We note that the transpose of the inverse of $B$ in (24) is the incidence matrix of $\sigma$. This identification is generally true for Rauzy induction, due to the identification of the $i$ th substitution string with the first-return itinerary of the $i$ th IET sub-interval. 
Finally, we verify that the drift vector is non-zero. The integer translation vectors $v_{i}=\phi\left(\tau_{i}\right)$ - see equation ([6) - are

$$
(1,-1,0,0),(1,-5,5,-1),(-1,3,-1,0),(0,-1,0,0),
$$

so that

$$
\mathcal{S}=\sum_{i=1}^{4} \Lambda_{i} v_{i}=\left(\rho-4 \rho^{2}-\rho^{3},-1+16 \rho^{2}-4 \rho^{3}, 4-16 \rho+\rho^{3},-1+4 \rho-\rho^{2}\right)
$$

is non-zero (since $\rho$ is an algebraic number of degree four).

The cycle of length 8 containing $E$ is the only one with a quartic irreducible polynomial. The other members of the cycle give 7 other IETs which renormalize with a scale factor $\rho$, but with different permutations and sets of widths in the ring $\mathbb{Z}[\rho]$. The example $E$ has the nice property that the rescaled interval is just $\Omega_{1}$, a feature not shared by all of its cycle-mates. For example, the cycle contains another IET with permutation (4213) for which the induced map again acts on the interval $(0, \rho), \rho \approx 0.227777$, but the latter is properly contained in $\Omega_{1}=[0, \mu), \mu=-1+7 \rho-6 \rho^{2}+\rho^{3} \approx 0.294963$.

Interestingly, for longer cycles we also have relatively few irreducible quartic polynomials (hence rings). The table below gives a summary of the numbers of suitable Rauzy cycles and associated polynomials for cycle length up to 16 .

\begin{tabular}{|c|c|c|}
\hline cycle length & $\sharp$ Rauzy cycles & $\sharp$ polys \\
\hline 8 & 1 & 1 \\
9 & 6 & 3 \\
10 & 7 & 4 \\
11 & 30 & 10 \\
12 & 80 & 27 \\
13 & 148 & 50 \\
14 & 360 & 108 \\
15 & 798 & 211 \\
16 & 1657 & 452 \\
\hline
\end{tabular}

\subsection{Zero drift-vector}

Here we are interested in IET belonging to the Arnoux-Rauzy family [6] to which belongs the Arnoux-Yoccoz example $[4,7]$ studied in great detail in $[18,19]$. Originally, this family was found as a way to construct a sequence of complexity $2 n+1$. The construction is the following (we keep the original notation from $[6]$ ).

Let $\alpha, \beta, \gamma$ be three positive real numbers such that $\alpha>\beta+\gamma$. We define a sixinterval-exchange map $f$ over the circle of length $\alpha+\beta+\gamma$ as follows. First, we divide 
in two halves the three intervals $I_{\alpha}, I_{\beta}, I_{\gamma}$ with respective lengths $\alpha, \beta, \gamma$ and we swap the halves preserving the orientation. Then we rotate the whole circle by $\pi$. It can then be shown than the induced map on $f\left(I_{\alpha}\right)$ has the same structure over three intervals of respective lengths $\alpha^{\prime}=\alpha-\beta-\gamma, \beta^{\prime}=\beta, \gamma^{\prime}=\gamma$. We can iterate this process: at each step we induce on the largest interval, and at each step one of the intervals of the induced map is larger than the sum of the other two. We can see that this process has the same flavour as Rauzy induction. If we define the three matrices

$$
M_{1}=\left(\begin{array}{lll}
1 & 1 & 1 \\
0 & 1 & 0 \\
0 & 0 & 1
\end{array}\right) \quad M_{2}=\left(\begin{array}{lll}
1 & 0 & 0 \\
1 & 1 & 1 \\
0 & 0 & 1
\end{array}\right) \quad M_{3}=\left(\begin{array}{lll}
1 & 0 & 0 \\
0 & 1 & 0 \\
1 & 1 & 1
\end{array}\right)
$$

then it can be shown that for any $k$ the product $M_{1} M_{2}^{k} M_{3}$ occurs infinitely many times in the possible sequences of induction. That is, for each $k$, the Perron eigenvector of each such product will give the lengths of a renormalizable map $E_{k}$ over the circle. By construction, all members of this family have zero Sah-Arnoux-Fathi invariant, and hence zero drift vector (proposition 5 ).

The case $k=1$ is the Arnoux-Yoccoz case with three intervals, with characteristic polynomial $p(x)=x^{3}-7 x^{2}+5 x-1$. Its unique real root is the scaling constant $\rho=\lambda^{-3}$, where $\lambda$ is the so-called tribonacci number [22, p. 233]. The lengths are $(\alpha, \beta, \gamma)=\left(\lambda^{-2}, \lambda^{-1}, 1\right)$. This example has very interesting properties, which include finite decomposition.

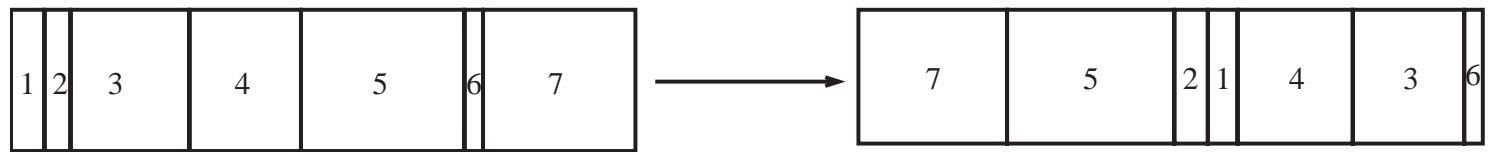

Figure 5: The interval exchange $E_{2}$.

For $k>1$, the maps maps $E_{k}$ are quite similar to the Arnoux-Yoccoz map. We will present some computations for the cases $k=2,3,4,5,6$ (see figure 5 for $k=2$ ), which show that they are likely to share the finite decomposition property.

The module of $E_{k}$ is the half-integer lattices $\mathcal{M}=\mathbb{Z}\left[\lambda_{k}\right] / 2$, where $\lambda_{k}$ is the smallest real roots of the polynomial $f_{k}$, given by

$$
f_{k}(x)=x^{3}-(k+4) x^{2}+(3 k+4) x-1, \quad k \geq 1 .
$$

One verifies that for all $k \geq 1$, the polynomial $f_{k}$ has no integer roots and hence is irreducible. The (non-normalized) vector of lengths of the partition is

$\Lambda=\left(\lambda_{k}-\lambda_{k}^{2} / 2, \lambda_{k}-\lambda_{k}^{2} / 2,1 / 2-3 \lambda_{k} / 2+\lambda_{k}^{2} / 2,1 / 2-3 \lambda_{k} / 2+\lambda_{k}^{2} / 2,1 / 2, \lambda_{k} / 2,1 / 2-\lambda_{k} / 2\right)$,

and the corresponding translation vectors are

$$
\frac{1}{2}\left(2+\lambda_{k}-\lambda_{k}^{2}, 2-3 \lambda_{k}+\lambda_{k}^{2}, 3-4 \lambda_{k}+\lambda_{k}^{2}, 1+2 \lambda_{k}-\lambda_{k}^{2}, \lambda_{k}-1,1-\lambda_{k}, \lambda_{k}-3\right) .
$$

One can then verify directly that the drift vector is indeed zero. The maps $E_{k}$ are not renormalizable but in each cases — as in [18] — the induced map in the first interval leads 
to a renormalizable IET on seven intervals. We shall carry out the computations directly with the maps $E_{k}$.

In order to investigate the finite decomposition property, we will perform a fairly straightforward algorithm implemented in C. For each $k$ we will first select the slab of the 3 -dimensional half-integer lattice consisting of the points belonging to the interval $\left[0,2-\lambda_{k}\right)$ which lie inside a cube of prescribed size. Then we iterate forward and backward all the points in a small cube around the origin to check how many points in the big cube slab are attained. This method is fast, easy to implement, and applicable to any algebraic IET. It is however memory-intensive, which constrained the maximum linear size of the cube we were able to consider to about 600 points. For the sake of speed, when mapping a lattice point into the intervals we use standard double precision (as opposed to arbitrary precision). It should be noted however that there is no error propagation in the dynamics, as each point is represented in vector form using the $\mathbb{Z}$-module basis.

To represent lattice points, we chose the basis $\left(1, \lambda_{k}, \lambda_{k}^{2}\right)$ for $\mathbb{Z}[\lambda]$. It should be noted that this choice is not necessarily the best in all cases. Indeed, orbits are often highly "anisotropic", escaping much faster in a specific direction than in others. As a consequence, covering a significant part of the cube around the origin could be time-consuming. While more favourable bases have been found in special cases, no general optimal family has been found. For instance, for $k=1$ we have the same case as in [18] and yet the orbits are not distributed as uniformly as in this paper. In fact, $\lambda_{1}$ is the third-root of the tribonacci number used as a basis in [18], switching to this basis gives more uniform orbits. This process does not generalize to greater $k$ though.

Nevertheless, on all the cases we have investigated we see that a very small set of initial conditions is required to cover at least a significant part of the lattice around zero. We define

$$
\mathcal{C}_{D}=\left\{\frac{1}{2}(p, q, r) \in \frac{1}{2} \mathbb{Z}^{3}: \frac{1}{2}\left(p+q \lambda_{k}+r \lambda_{k}^{2}\right) \in\left[0,2-\lambda_{k}\right), \quad \max \{|p|,|q|,|r|\} \leq 2 D\right\} .
$$

In table1, we summarize the results of the investigations. For each $k$, we give the maximum size $D$ of $\mathcal{C}_{D}$ that we were able to fill with backward and forward iterates of points in an initial region $\mathcal{C}_{d}$ of size $d$. We also give an upper bound on the number $T$ of iterations required.

\begin{tabular}{|c|c|c|c|}
\hline$k$ & $D$ & $d$ & $T$ \\
\hline 2 & 400 & 1 & $3 \times 10^{6}$ \\
3 & 400 & 3 & $6 \times 10^{7}$ \\
4 & 400 & 1 & $2.6 \times 10^{8}$ \\
5 & 400 & 1 & $9 \times 10^{8}$ \\
6 & 200 & 3 & $1 \times 10^{9}$ \\
\hline
\end{tabular}

Table 1: Data for lattice-filling experiments.

For each $k$, the substitutions $\sigma_{k}$ are explicitly computable and we can use propositions 10 and 11 to give us additional evidence regarding the finite-decomposition hypothesis. Indeed the latter proposition gives us asymptotic estimates on the escape rate of orbits 
of the lattices having periodic Vershik code, while the former gives a condition for these orbits to be fairly common. Both proposition rely on the value of the following quantity

$$
v=\frac{\log \|R\|}{\log \left\|M_{\sigma_{k}}\right\|}
$$

which gives the exponent of the escape rate of the orbit of a point having periodic Vershik code. These points have density one in at least one coset $\xi+\mathcal{M}$ if $v=1 /(n-1)$. In the following table we give approximations of the values of $v$ for several values of $k$

\begin{tabular}{|c|c|c|c|c|c|c|c|}
\hline$k$ & 1 & 2 & 3 & 4 & 5 & 6 & 7 \\
\hline$v$ & 0.5 & 0.5 & 0.5 & 0.546715 & 0.595958 & 0.623202 & 0.642502 \\
\hline
\end{tabular}

We see that for $k=1,2,3$, we can deduce from the proposition mentioned above that the points having periodic Vershik code (a set which have positive density) have positive density, which is another indication that $E_{2}, E_{3}$ might have the finite decomposition property. The data for the other values of $k$ are inconclusive though. The following table shows values of $v$ for increasing values of $k$. The value of $v$ appears to increases slowly toward unity.

\begin{tabular}{|c|c|c|c|c|c|c|c|}
\hline$k$ & 17 & 30 & 50 & 100 & 150 & 200 & 500 \\
\hline$v$ & 0.721655 & 0.756235 & 0.780871 & 0.8074 & 0.820165 & 0.828247 & 0.849766 \\
\hline
\end{tabular}

This can be explained if we look at the partitions of the maps $E_{k}$ for increasing $k$. As displayed in figure (6), we see that the intervals carrying the "rotation-flip" dynamics tends to fill the whole interval, leading to a global dynamics approaching a simple non-zero drift dynamics, for which $v=1$.

\subsection{Self-similar $E_{2}$ model}

We conclude our discussion of zero-drift-vector examples with a more detailed look at a self-similar version of the map $E_{2}$ introduced in section 4.2. The particular IET, which we shall call $E_{2}^{*}$, is a member of a 29-vertex cycle in the same 294-vertex Rauzy class of 7-interval IETs as the self-similar Arnouz-Yoccoz model of [18]. Its permutation is (5462731), and the 7-dimensional cyclic product matrix is

$$
B=\left(\begin{array}{lllllll}
4 & 9 & 6 & 6 & 4 & 8 & 2 \\
0 & 2 & 1 & 1 & 0 & 1 & 0 \\
0 & 2 & 3 & 0 & 2 & 2 & 0 \\
1 & 2 & 1 & 2 & 0 & 1 & 0 \\
1 & 1 & 1 & 1 & 2 & 2 & 1 \\
0 & 2 & 2 & 0 & 2 & 3 & 0 \\
1 & 1 & 1 & 1 & 1 & 1 & 1
\end{array}\right)^{-1}
$$

with characteristic polynomial (cf. equation (20)) )

$$
P(x)=\left(x^{3}-6 x^{2}+10 x-1\right)\left(x^{3}-10 x^{2}+6 x-1\right)(1-x) .
$$



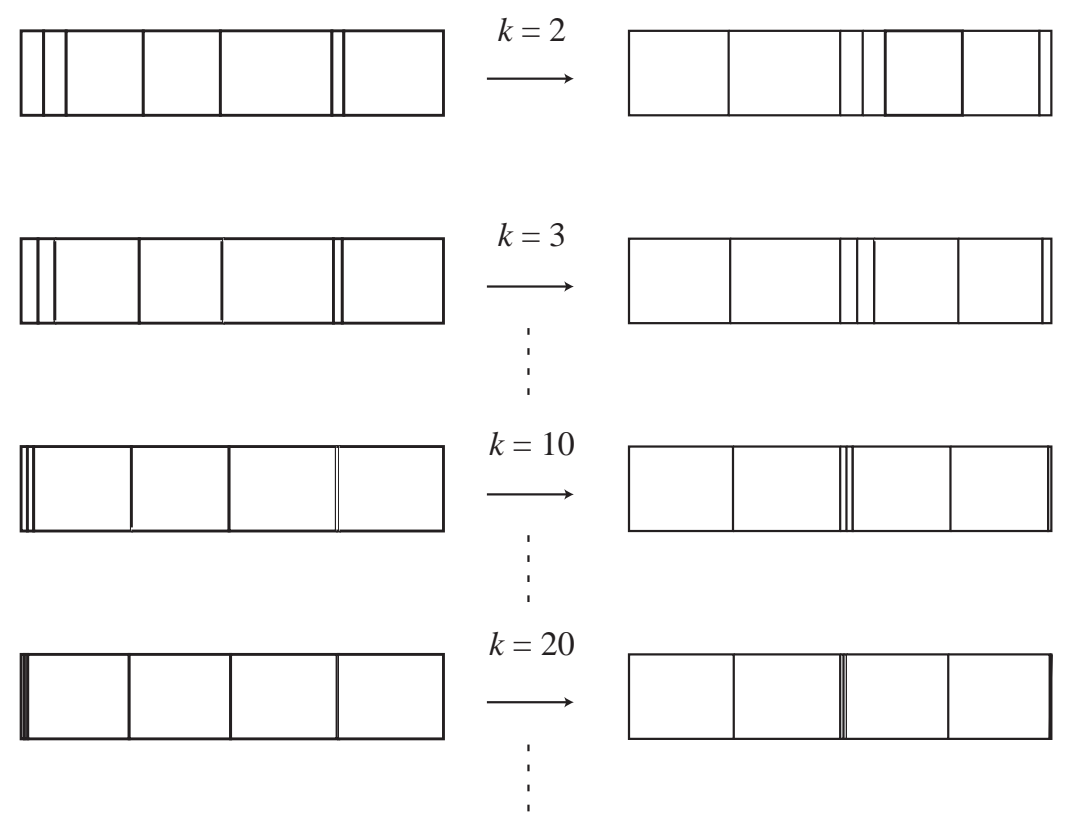

Figure 6: The interval exchanges $E_{k}$ for increasing $k$, showing that three intervals tends to vanish, and the dynamics approaches a rotation with flips.

The scaling constant $\rho \approx 0.106711$ is the real root of the polynomial

$$
p(x)=x^{3}-6 x^{2}+10 x-1 .
$$

One verifies that $\beta=\rho^{-1}$, the real root of $\tilde{p}(x)=x^{3}-10 x^{2}+6 x-1$, is a Pisot number.

The 29 members of the Rauzy cycle containing $E_{2}^{*}$ are all self-similar IETs with the same scale factor $\rho$. The map $E_{2}^{*}$ was selected for special consideration because of several additional simplifying features. In particular, its translation module is $\mathcal{M}=\mathbb{Z}[\rho]$, its rightmost interval $\Omega_{7}$ is of length $\rho$, and the induced IET on $\Omega_{7}$ is just the original map rescaled by $\rho$.

The vector $\Lambda$ of interval lengths is the positive 7-vector associated with $\rho$ in the diagonalization of $B^{-1}$, normalized to total length unity. Specifically, choosing the canonical basis $\left(1, \rho, \rho^{2}\right)$ for $\mathcal{M}=\mathbb{Z}[\rho]$, we find

$$
\Lambda=\left(1-5 \rho+2 \rho^{2},-1+10 \rho-3 \rho^{2}, 1-9 \rho+3 \rho^{2}, \rho-\rho^{2},-1+11 \rho-4 \rho^{2}, 1-9 \rho+3 \rho^{2}, \rho\right)
$$

with corresponding integers translations $v_{i}=\phi\left(\tau_{i}\right), i=1, \ldots, 7$,

$$
\left(v_{i}\right)=((0,3,-1),(0,-2,0),(1,-7,2),(-1,5,-2),(1,-8,3),(0,-6,2),(-1,1,0)) .
$$

The IET is illustrated in figure 7 Calculating $\sum_{i} \Lambda_{i} v_{i}$, we verify directly that the drift vector vanishes.

Iterating the map $E_{2}^{*}$, we can examine the first-return orbits of the 7 intervals of the induced map on $\Omega_{7}$ and establish the recursive tiling property, with associated substitution 


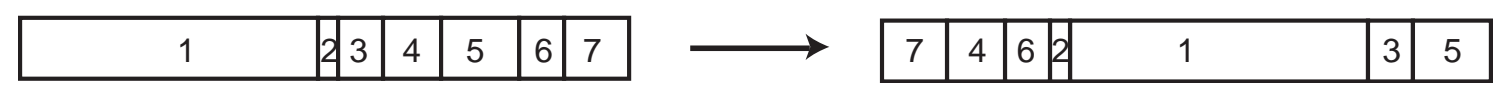

Figure 7: The self-similar $E_{2}$ map.

rule

$$
\sigma:\left\{\begin{array}{lll}
1 & \longrightarrow & 7114115 \\
2 & \longrightarrow & 7114121361361214115 \\
3 & \longrightarrow & 711412136136135 \\
4 & \longrightarrow & 71141214115 \\
5 & \longrightarrow & 71156136135 \\
6 & \longrightarrow & 711561361361214115 \\
7 & \longrightarrow & 7115
\end{array}\right.
$$

We obtain the following result:

Proposition 13 We consider the mapping $E_{2}^{*}$. The following holds

i) Let $x$ be a point of the unit interval and let $\mu(x)$ be its Vershik code. Then $x \in$ $\mathbb{Q}(\rho) \cap[0,1)$ if and only if $\mu(x)$ is eventually periodic.

ii) The restriction of the module $\mathcal{M}$ to the unit interval consists of the (forward and backward) orbits of the discontinuity points.

iii) The map has the finite decomposition property. Specifically, there is a positive integer $M$ such that for each $\xi \in K$ the restriction of the layer $\xi+\mathcal{M}$ to the unit interval decomposes into at most $M$ orbits.

We shall not provide a detailed proof of the proposition above, since it is a straightforward adaptation to the present model of the proof provided in section 3 of [18] for the Arnoux-Yoccoz cubic model. Let us merely summarize the main ideas. We represent a point $x$ in $\mathbb{Q}(\rho) \cap[0,1)$, as $x=\xi+z$, with $\xi \in \Xi$ - see equation (13) - and $z \in \mathbb{Z}[\rho]$. Specifically, using the basis $\left(1, \rho, \rho^{2}\right)$ for $\mathbb{Q}(\rho)$ we have

$$
\begin{array}{rlc}
\xi & =r_{0}+r_{1} \rho^{2}+r_{2} \rho^{2} & r_{i} \in \mathbb{Q} \cap[0,1) \\
z & =m_{0}+m_{1} \rho+m_{2} \rho^{2} & m_{i} \in \mathbb{Z} .
\end{array}
$$

The restriction of $x$ to the unit interval leads to the condition

$$
m_{0}=-\left\lfloor\xi+m_{1} \rho+m_{2} \rho^{2}\right\rfloor .
$$

Any point on the forward or backward orbit of $x$ can be represented in the same way, with the same $\xi$. Since each point is completely determined by an integer pair $\left(m_{1}, m_{2}\right)$, it is natural to associate the orbit with a walk on the lattice $\mathbf{L}_{\xi}^{\prime} \cong \mathbb{Z}^{2}$ (with reference to equation (9), here we have $n=3$ and $b=1$ ). 
To prove the finite decomposition property of proposition [13, we must show that the number of lattice orbits in $\mathbf{L}_{\xi}^{\prime}$ is bounded uniformly in $\xi$. The strategy is to show that every lattice orbit visits a finite $\xi$-independent core region containing no more than a finite number $M$ of lattice points. The existence of such a region is also the key to proving the second statement in proposition 13 , one needs only to verify that the lattice orbits of the discontinuity points visit all of the points of the core region of $\mathbf{L}_{0}^{\prime}$.

To complete the proof, one shows that for each $\xi \in \Xi$, some $k$ th iterate of the left-shift map for the Vershik code is represented in $\mathbf{L}_{\xi}^{\prime}$ by a map $\gamma^{k}$ with the following property: every $\left(m_{1}, m_{2}\right) \in \mathbf{L}_{\xi}^{\prime}$ has a forward $\gamma^{k}$ orbit which is eventually trapped in a finite, $\xi$ independent core region. This orbit must eventually settle down in a limit cycle of some finite period $t$. Thus the original point $x$ has an eventually periodic Vershik code of period $k t$, thereby establishing the "only if" part of proposition $13 \mathrm{i}$ ); while the "if" part follows at once from (22). Since the periodic tail of this code coincides with that of a point in the limit cycle in the core region, the two points lie on the same $E_{2}^{*}$ orbit, and we have the remaining element in the proof of the finite decomposition property as well.

It is important to point out that the proof above relies essentially on the self-similarity of the IET, and on the Pisot property of the reciprocal of the scale factor. In particular, it is the latter which ensures a contraction to the core region. In [18], additional properties of the specific IET were used to characterize in some detail the lattice orbits in the various $\mathbf{L}_{\xi}^{\prime}$. The orbits of the self-similar Arnoux-Yoccoz model were found to be non-crossing, filling out with unit density disjoint sectors of the lattices. It turns out that the lattice orbits of the $E_{2}^{*}$ model do not have these features. This is already true for the discontinuity-point orbits, for which the lattice is partitioned into three sectors, each filled out by a pair of mutually crossing orbits, neither of which is locally lattice-filling. The situation near the origin is shown in figure 8 ,

\section{References}

[1] B. Adamczewski, Symbolic discrepancy and self-similar dynamics, Ann. Inst. Fourier 54 (2004) 2201-2234.

[2] O. Ahmadi and G. Vega, On the parity of the number of irreducible factors of selfreciprocal polynomials over finite fields, Technical Report, CACR 10 (2006).

[3] A. Avila, G. Forni, Weak mixing for interval exchange transformations and translation flows, to appear in Annals of Mathematics.

[4] P. Arnoux, Un exemple de semi-conjugaison entre un change d'intervalles et une translation sur le tore, Bull. Soc. Math. France 116 no. 4 (1989) 489-500.

[5] P. Arnoux, Un invariant pour les échanges d'intervalles et les flots sur les surfaces, PhD thesis, Université de Reims (1981).

[6] P. Arnoux, G. Rauzy, Représentation géométrique de suites de complexité $2 n+1$, Bull. Soc. Math. France 119 no. 2 (1991) 199-215. 


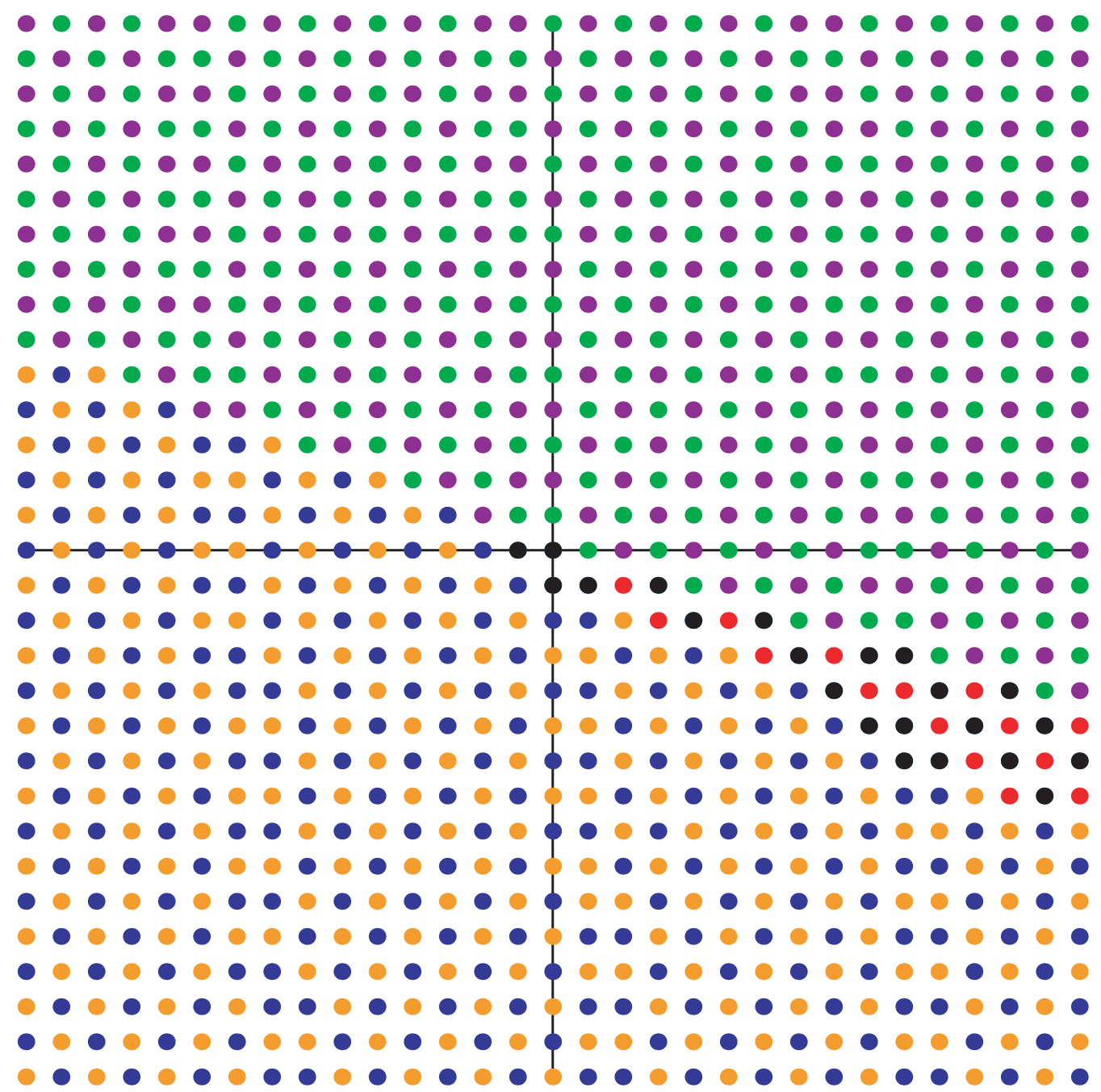

Figure 8: Points of the six lattice orbits of the discontinuity points of the IET $E_{2}^{*}$. Each of the orbits is arbitrarily assigned a colour.

[7] P. Arnoux and J. Yoccoz, Construction de diffeomorphismes pseudo-Anosov, C. R. Acad. Sci. Paris 292 (1981) 75-78.

[8] M. Boshernitzan, A condition for minimal interval exchange map to be uniquely ergodic, Duke Math. J. 52 no. 3 (1985) 723-752.

[9] M. D. Boshernitzan and C. R. Carroll, An extension of Lagrange's theorem, J. d'Analyse Math. 72 (1997) 21-44.

[10] H. Cohen, A course in computational algebraic number theory, Springer-Verlag, Berlin (1996).

[11] N. Jacobson, Basic Algebra I, W. H. Freeman, New York (1985).

[12] A. B. Katok, Interval exchange transformations and some special flows are not mixing, Israel J. Math. 35 (1980) 301-310. 
[13] M. Keane, Interval exchange transformations, Math. Zeitsch. 141 (1975) 25-31.

[14] K. L. Kouptsov, J. H. Lowenstein and F. Vivaldi, Quadratic rational rotations of the torus and dual lattice maps, Nonlinearity 15 (2002) 1795-1842.

[15] J. S. W. Lamb and J. A. G. Roberts, Time-reversal symmetry in dynamical systems: A survey, Physica D 112 (1998) 1-39.

[16] J. H. Lowenstein, K. L. Kouptsov, and F. Vivaldi, Recursive tiling and geometry of piecewise rotations by $\pi / 7$, Nonlinearity 17 (2004) 1-25.

[17] J. H. Lowenstein, G. Poggiaspalla, and F. Vivaldi, Sticky orbits in a kicked-oscillator model, Dynamical Systems, 20 no.4 (2005) 413-451.

[18] J. H. Lowenstein, G. Poggiaspalla, and F. Vivaldi, Interval exchange transformations over algebraic number fields: the cubic Arnoux-Yoccoz model, Dynamical Systems 22 (2007) 73-106.

[19] J. H. Lowenstein and F. Vivaldi, Scaling dynamics of a cubic interrval-exchange transformation, submitted to Dynamical Systems (2007). http://www.maths.qmul.ac.uk/ fv/research/ScalingAY.pdf

[20] H. Masur, Interval exchange transformations and measured foliations, Annals of Mathematics 115 (1982) 169-200.

[21] G. Poggiaspalla, Self-similarity in piecewise isometric systems, Dyn. Syst., 21 no. 2 (2006) 147-189.

[22] N. Pytheas Fogg, Substitutions in dynamics, Arithmetics and Combinatorics, Springer-Verlag, Berlin (2002).

[23] G. Rauzy, Echanges d'intervalles et transformations induites, Acta Arith. 34 (1979) $315-328$.

[24] D. Roy and M. Waldschmidt, Simultaneous approximation and algebraic independence, The Ramanujan Journal 4 (1997) 379-430.

[25] W. Veech, Gauss measures for transformations on the space of interval exchange maps Ann. of Math., 115 (1982) 201-242.

[26] W. Veech, The metric theory of interval exchange transformations III. The SahArnoux-Fathi invariant Amer. J. Math., 106 no. 6 (1984) 1389-1422.

[27] A. Zorich, Deviation for interval exchange transformations, Ergod. Th. and Dynam. Sys., 17 (1997) 1477-1499.

[28] A. Zorich, Finite Gauss measure on the space of interval exchange transformations. Lyapunov exponents, Annales de l'Institut Fourier 46:2 (1996) 325-370. 